W. H. Fleming and M. Nisio

Nagoya Math. J.

Vol. 93 (1984), 71-108

\title{
ON STOCHASTIC RELAXED CONTROL FOR PARTIALLY OBSERVED DIFFUSIONS
}

\author{
W. H. FLEMING AND M. NISIO
}

\section{§. Introduction}

In this paper we are concerned with stochastic relaxed control problems of the following kind. Let $X(t), t \geq 0$, denote the state of a process being controlled, $Y(t), t \geq 0$, the observation process and $p(t, \cdot)$ a relaxed control, that is a process with values probability measures on the control region $\Gamma$. The state and observation processes are governed by stochastic differential equations

$$
\left\{\begin{array}{l}
d X(t)=\alpha(X(t)) d B(t)+\int_{\Gamma} r(X(t), u) p(t, d u) d t \\
X(0)=\xi
\end{array}\right.
$$

and

$$
\left\{\begin{array}{l}
d Y(t)=h(X(t)) d t+d W(t) \\
Y(0)=0
\end{array}\right.
$$

where $B$ and $W$ are independent Brownian motions with values in $R^{n}$ and $R^{m}$ respectively, (put $m=1$ for simplicity).

The problem is to maximize a criterion of the form

$$
J=E f(X(T))
$$

by a suitable choice of admissible relaxed control $p$. In a customary version of stochastic control under partial observation, $p(t, \cdot)$ is measurable with respect to $\sigma$-field generated by the observation process $Y(s)$, $s \leq t$. Instead of discussing the problem of this type, we treat some wider class of admissible relaxed controls (see $\S 2$ ), inspired by FlemingPardoux [8]. Roughly speaking, our problem is the following; Let

Received September 13, 1982.

This research was supported in part by the National Science Foundation under contract MCS 79-03554 and in part by the Air Force Office of Scientific Research under contract AF-AFOSR 81-0116. 


$$
L(T)=\exp \left\{\int_{0}^{T} h(X(s)) d Y(s)-\frac{1}{2} \int_{0}^{T}|h(X(s))|^{2} d s\right\}
$$

Then $B$ and $Y$ turn out as independent Brownian motions under a new probability $\stackrel{\circ}{P}$, defined by

$$
\frac{d \stackrel{P}{d P}}{d P} L^{-1}(T)
$$

appealing to the so-called Girsanov transformation. For admissibility we merely require that $p(t, \cdot)$ is independent of future increments of $Y(\theta)-$ $Y(s), \theta, s \geq t$, and $B$, with respect to $\stackrel{\circ}{P}$. Moreover we are concerned with $q(d t, d u)$ instead of $p(t, d u) d t$. (see Definition 1). Thus the criterion $J$ can be expressed as

$$
J=\stackrel{\circ}{E} f(X(T)) L(T)
$$

where $\stackrel{\circ}{E}$ stands for the expectation with respect to $\stackrel{\circ}{P}$, and $X(t)$ is a solution of the following system equation;

$$
d X(t)=\alpha(X(t)) d B(t)+\int_{\Gamma} \gamma(X(t), u) q(d t, d u) .
$$

Under Lipschitz continuity and boundedness of $\alpha$ and $\gamma,(1.6)$ has a unique solution (Theorem 1).

In Section 2 we introduce some metric spaces which are appropriate to our optimization problems. In Section 3 we prove the compactness of spaces of solutions and relaxed controls $q$. This guarantees the existence of optimal one (Theorem 3).

In the latter half we treat a nonlinear semigroup associated with relaxed control under partial observation. In this case we regard, as the state space, the unnormalized conditional distribution $\Lambda(t)$ of $X(t)$ given past observation and control. Hence $\Lambda(t)$ is a process valued in measures on $R^{n}$ and satisfying the Zakai equation. Thus our problems turn out as optimization problems of measure valued processes. After we prove the continuity of $\Lambda(t)$ with respect to initial distribution $X(0)$ and data of past observation and control, (see Theorem 5), we construct a nonlinear semigroup $S(t), t \geq 0$, on a Banach lattice of bounded and uniformly continuous functions, defined on the space of measures (Theorem 7). Following Fleming [6], we show that the generator of $S(t)$ relates to a dynamic programming equation, so-called Mortensen's equation. 


\section{§2. Notations and preliminaries}

Let $(\Omega, F, \stackrel{\circ}{P})$ be a probability space. Let $B$ and $Y$ be $n$-dimensional and 1-dimensional Brownian motions, defined on $(\Omega, F, \stackrel{P}{)})$ respectively. $\Gamma$ is a given convex compact subset of $R^{k}$, called a control region. $M(\Gamma)$ denotes the totality of positive finite measures defined on $B(\Gamma)$ (=Borel field of $\Gamma)$. By $\hat{M}\left(\left[\begin{array}{ll}0 & T\end{array}\right] \times \Gamma\right)$ we denote the set of all mappings $\lambda$ : $\left[\begin{array}{ll}0 & T\end{array}\right]$ $\times B(\Gamma) \rightarrow\left[\begin{array}{ll}0 & T\end{array}\right]$ such that

0) $\lambda(0, A)=0 \quad \forall A \in B(\Gamma)$

i ) $\lambda(t, \Gamma)=t \quad \forall t \geq 0$

ii) $\lambda(t, \cdot) \in M(\Gamma)$ for all $t>0$

iii) $\lambda(t, A)$ is increasing in $t$ for all $A \in \boldsymbol{B}(\Gamma)$

iv) $\sup _{A \in B(\Gamma)}|\lambda(s, A)-\lambda(t, A)|=|t-s|$.

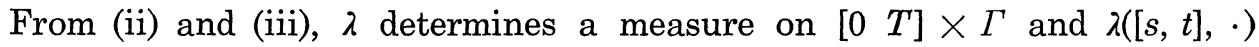
$\equiv \lambda(t, \cdot)-\lambda(s, \cdot) \in M(\Gamma)$, if $t>s$, and $\lambda([s, t], \Gamma)=t-s$.

Let $q$ be a mapping; [0 $T] \times \boldsymbol{B}(\Gamma) \times \Omega \rightarrow[0,1]$, such that

v) for all $A \in B(\Gamma), q(\cdot, A, \cdot)$ is $B[0 T] \times F$-measurable

vi) $q \in \hat{M}\left(\left[\begin{array}{ll}0 & T\end{array}\right] \times \Gamma\right) \stackrel{\circ}{P}$-almost surely.

Definition 1. $\mathscr{A}=(\Omega, F, \stackrel{\circ}{P}, \xi, B, Y, q)$ is called an admissible (relaxed) system, if $\xi$ is an $n$-random vector on $(\Omega, F, \stackrel{\circ}{P})$, which is independent of $(B, Y, q), B$ and $(Y q)$ are independent and the increments $(Y(t)-$ $Y(s), t \geq s)$ are independent of $\sigma_{s}(Y, q)(=\sigma$-field generated by $Y(\theta), \theta \leq s$ and $q(\theta, A), \theta \leq s, A \in \boldsymbol{B}(\Gamma))$.

Definition 2. The component $q$ of $\mathscr{A}$ is called a relaxed control, and we denote it by $q_{\&}$ when $\mathscr{A}$ is stressed. $\lambda \in \hat{M}\left(\left[\begin{array}{ll}0 & T\end{array}\right] \times \Gamma\right)$ can be regarded as a relaxed control. $\mathfrak{A}$ denotes the totality of admissible systems.

Let $\alpha(x)$ be a symmetric $n \times n$ matrix valued function on $R^{n}$ and $\gamma$ an $n$-vector continuous function on $R^{n} \times \Gamma$. We assume the following conditions

(A1) $|g(x, u)| \leq b, \quad \forall x \in R^{n}, u \in \Gamma g=\alpha, \gamma$

(A2) $\left|g(x, u)-g\left(x^{\prime}, u\right)\right| \leq K\left|x-x^{\prime}\right|, \forall x, x^{\prime} \in R^{n} u \in \Gamma, g=\alpha, \gamma$.

For an admissible system $\mathscr{A}=(\Omega, F, \stackrel{\circ}{P}, \xi, B, Y, q)$ we consider the stochastic differential equation (SDE in short)

$$
\left\{\begin{array}{l}
d X(t)=\alpha\left(X(t) d B(t)+\int_{\Gamma} \gamma(X(t), u) q(d t, d u)\right. \\
X(0)=\xi
\end{array}\right.
$$


TheOREM 1. There exists a unique solution $X$ of (2.1) which is $\sigma_{t}(\xi, B, q)$-progressively measurable and has continuous paths.

Proof. We apply a usual successive approximation method. We define $X_{n}$ in the following way

$$
X_{0}(t)=\xi
$$

$$
\begin{array}{r}
X_{n+1}(t)=\xi+\int_{0}^{t} \alpha\left(X_{n}(s)\right) d B(s)+\int_{0}^{t} \int_{\Gamma} \gamma\left(X_{n}(s), u\right) q(d s, d u) \\
n=0,1,2, \cdots
\end{array}
$$

Then, $X_{n}$ is $\sigma_{t}(\xi, B, q)$-progressively measurable and has continuous paths by (iv) and (A1).

$$
\begin{aligned}
X_{n+1}(t)-X_{n}(t)=\int_{0}^{t}( & \left.\alpha\left(X_{n}(s)\right)-\alpha\left(X_{n-1}(s)\right) d B(s)\right) \\
& \quad+\int_{0}^{t} \int_{\Gamma}\left(\gamma\left(X_{n}(s), u\right)-\gamma\left(X_{n-1}(s), u\right)\right) q(d s, d u)
\end{aligned}
$$

So, using (A1) and (A2) we see

$$
\begin{aligned}
& \left(\int_{0}^{t} \int_{\Gamma}\left|\gamma\left(X_{n}(s), u\right)-\gamma\left(X_{n-1}(s), u\right)\right| q(d s, d u)\right)^{2} \\
& \quad \leq \int_{0}^{t} \int_{\Gamma}\left|\gamma\left(X_{n}(s), u\right)-\gamma\left(X_{n-1}(s), u\right)\right|^{2} q(d s, d u) q(t, \Gamma) \\
& \quad \leq K t \int_{0}^{t} \int_{\Gamma}\left|X_{n}(s)-X_{n-1}(s)\right|^{2} q(d s, d u) \\
& \quad=K t \int_{0}^{t}\left|X_{n}(s)-X_{n-1}(s)\right|^{2} d s
\end{aligned}
$$

Putting $\rho_{n}(t)=E\left|X_{n+1}(t)-X_{n}(t)\right|^{2}$, we have

$$
\rho_{n}(t) \leq K_{1} \int_{0}^{t} \rho_{n-1}(s) d s, \quad \text { for } \forall t \leq T,
$$

with some $K_{1}=K_{1}(T)$. This implies

$$
\rho_{n}(t) \leq \frac{t^{n-1} K_{1}^{n-1}}{(n-1) !} \stackrel{\circ}{E}|\xi|^{2} .
$$

Therefore

$$
\sum_{n} \stackrel{\circ}{E}\left|X_{n+1}(t)-X_{n}(t)\right| \leq \sum \sqrt{\rho_{n}(t)}<\infty .
$$

This implies that $X_{n}(t)$ converges $\stackrel{\circ}{P}$-almost surely. Hence $X(t)=\lim _{n \rightarrow \infty} X_{n}(t)$ 
can be regarded as $\sigma_{t}(\xi, B, q)$-progressively measurable and moreover a martingale inequality tells us that, as $n \rightarrow \infty$,

$$
\int_{0}^{t} \alpha\left(X_{n}(s)\right) d B(s) \rightarrow \int_{0}^{t} \alpha(X(s)) d B(s) \text { uniformly in } t \in[0, T],
$$

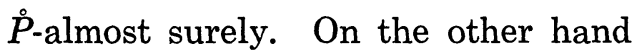

$$
\begin{gathered}
\sup _{t \leq T} \mid \int_{0}^{t} \int_{\Gamma} \gamma\left(X_{n}(s), u\right)-\gamma(X(s), u) q(d s, d u) \\
\quad \leq \int_{0}^{T} \int_{\Gamma}\left|\gamma\left(X_{n}(s), u\right)-\gamma(X(s), u)\right| q(d s, d u) \\
\quad \leq K \int_{0}^{T} \min \left(\left|X_{n}(s)-X(s)\right|, 2 b\right) d s .
\end{gathered}
$$

By virtue of the convergence theorem we get, as $n \rightarrow \infty$,

$$
\int_{0}^{t} \int_{\Gamma} \gamma\left(X_{n}(s), u\right) q(d s, d u) \rightarrow \int_{0}^{t} \int_{\Gamma} \gamma(X(s), u) q(d s, d u),
$$

uniformly in $t \in[0, T], \stackrel{\circ}{P}$-almost surely.

Combining (2.4) and (2.5) with (2.2), $X$ turns out as a solution of (2.1) and $X_{n}(t)$ converges to $X(t)$ uniformly in $t \in\left[\begin{array}{ll}0 & T\end{array}\right] \stackrel{\circ}{P}$-almost surely. Hence $X$ has continuous paths.

Let $Y$ be a $\sigma_{t}(\xi, B, q)$-progressively measurable solution of (2.1). Then, applying a routine method, we can easily see for ${ }^{\forall} t$,

$$
X(t)=Y(t) \quad \stackrel{\circ}{P} \text {-almost surely. }
$$

This completes the proof of Proposition 2.1.

$L$ denotes the Prohorov metric for probability measures. That is following, [11]. Let $\varepsilon_{21}$ be the infinimum of $\varepsilon$ such that

$$
\mu_{1}(F) \leq \mu_{2}\left(U_{s}(F)\right)+\varepsilon \quad \text { for all closed subset } F,
$$

where $U_{\varepsilon}(F)$ is the $\varepsilon$-neighbourhood of $F$. $\varepsilon_{12}$ is defined by switching $\mu_{1}$ and $\mu_{2}$. Set

$$
L\left(\mu_{1}, \mu_{2}\right)=\max \left(\varepsilon_{12}, \varepsilon_{21}\right)
$$

Put

$$
M(\Gamma, t)=\{\lambda \in M(\Gamma) ; \lambda(\Gamma)=t\}, t>0 .
$$

Define a metric $\rho_{t}$ as follows

$$
\rho_{t}\left(\lambda_{1}, \lambda_{2}\right)=L\left(\frac{\lambda_{1}}{t}, \frac{\lambda_{2}}{t}\right), \quad \lambda_{i} \in M(\Gamma, t) .
$$


Since $\Gamma$ is compact, $\left(M(\Gamma, t), \rho_{t}\right)$ is a compact metric space. Put $D=\left\{r_{i}\right.$, rational $\in[0, T], i=1,2, \cdots\}$ and

$$
\tilde{M}_{T}=\prod_{i=1}^{\infty} M\left(\Gamma, r_{i}\right)
$$

We endow $\tilde{M}_{T}$ with a metric $\tilde{d}_{T}$ such that

$$
\tilde{d}_{T}(\tilde{\lambda}, \tilde{\mu})=\sum_{n=1}^{\infty} \frac{1}{2^{n}} \rho_{r_{n}}\left(\lambda_{n}, \mu_{n}\right)
$$

where $\tilde{\lambda}=\left(\lambda_{1}, \lambda_{2}, \ldots\right)$ and $\tilde{\mu}=\left(\mu_{1}, \mu_{2}, \ldots\right)$. Hence $\tilde{\lambda}_{k}, k=1,2, \ldots$ is a $\tilde{d}_{T^{-}}$ Cauchy sequence, iff $\lambda_{k, i}, k=1,2, \ldots$ is a $\rho_{r_{i}}$-Cauchy sequence for any component $i$. Therefore again $\left(\tilde{M}_{T}, \tilde{d}_{T}\right)$ is a compact metric space.

Since $\lambda \in \tilde{M}\left(\left[\begin{array}{ll}0 & T\end{array}\right] \times \Gamma\right)$ is determined by $\tilde{\lambda}=\left(\lambda\left(r_{1}\right), \lambda\left(r_{2}\right), \cdots\right) \in \tilde{M}_{T}$, we define a metric $\hat{d}_{T}$ on $\hat{M}\left(\left[\begin{array}{ll}0 & T\end{array}\right] \times \Gamma\right)$ by

$$
\hat{d}_{T}(\lambda, \mu)=\tilde{d}_{T}(\tilde{\lambda}, \tilde{\mu}) .
$$

Proposition 2.1. $\hat{M}\left(\left[\begin{array}{ll}0 & T\end{array}\right] \times \Gamma, \hat{d}_{T}\right)$ is a compact metric space.

Proof. Let $\lambda_{k}\left(r_{i}, \cdot\right)$ converge to $\lambda_{(i)}$ in $\rho_{r_{i}}$. Then $\lambda_{(i)} \in M\left(\Gamma, r_{i}\right)$ and for $g \in C_{b}(\Gamma)$ (=) bounded continuous function on $\Gamma$ ).

$$
\int_{\Gamma} g(u) \lambda_{k}\left(r_{i}, d u\right) \rightarrow \int g(u) \lambda_{(i)}(d u), \quad \text { as } k \rightarrow \infty .
$$

Define $\lambda\left(r_{i}, A\right)$ by $\lambda\left(r_{i}, A\right)=\lambda_{(i)}(A)$. Then putting $g=1$ in (2.12), we see

$$
\lambda\left(r_{i}, \Gamma\right)=r_{i} .
$$

Let $r_{i}>r_{j}$ and set $R(A)=\lambda\left(r_{i}, A\right)-\lambda\left(r_{j}, A\right)$. Then $R$ is a signed measure on $\Gamma$. Since $\lambda_{k}\left(r_{i}, \cdot\right)-\lambda_{k}\left(r_{j}, \cdot\right) \in M\left(\Gamma, r_{i}-r_{j}\right)$ and $\lambda_{k}\left(r_{i}, \cdot\right)-\lambda_{k}\left(r_{j}, \cdot\right)$ converges to $R$ weakly by (2.12). $R$ turns out as a positive measure and $R \in M\left(\Gamma, r_{i}-r_{j}\right)$. This means

$$
\lambda(r, A) \text { is increasing in rational } r
$$

and

$$
\left|\lambda\left(r_{i}, A\right)-\lambda\left(r_{j}, A\right)\right| \leq\left|\lambda\left(r_{i}, \Gamma\right)-\lambda\left(r_{j}, \Gamma\right)\right|=r_{i}-r_{j} .
$$

Now we will construct $\lambda$ which corresponds to $\left(\lambda\left(r_{i}, \cdot\right), i=1,2, \cdots\right) \epsilon$ $\tilde{M}_{T}$, in the following way,

$$
\lambda(t, A)=\lim _{r_{i} \uparrow t} \lambda\left(r_{i}, A\right)
$$


Then $\lambda$ clearly satisfies the conditions (0) (iv), namely $\lambda \in M\left(\left[\begin{array}{ll}0 & T\end{array}\right] \times \Gamma\right)$ and $\hat{d}_{T}\left(\lambda_{k}, \lambda\right) \rightarrow 0$, as $k \rightarrow \infty$.

Remark. $\rho_{t}\left(\lambda_{k}(t), \lambda(t)\right) \leq\left(4 / r_{i}\right)\left|t-r_{i}\right|+\rho_{r_{i}}\left(\lambda_{k}\left(r_{i}\right), \lambda\left(r_{i}\right)\right)$ by condition (iv). Hence

$$
\lambda_{k}(t) \rightarrow \lambda(t) \quad \text { in } \rho_{t} .
$$

For $g \in C\left(\left[\begin{array}{ll}0 & T\end{array}\right] \times \Gamma\right)\left(=\right.$ continuous function on $\left.\left[\begin{array}{ll}0 & T\end{array}\right] \times \Gamma\right)$

$$
\int_{0}^{t} g(s, u) \lambda_{k}(d s, d u) \rightarrow \int_{0}^{t} g(s, u) \lambda(d s, d u), \quad \text { as } k \rightarrow \infty .
$$

Proof of (2.16). Since $g$ is uniformly continuous, $g\left(\left[2^{n} t\right] / 2^{n}, u\right)$ converges to $g(t, u)$ uniformly on $[0 T] \times \Gamma$, where $[c]$ is the largest integer $\leq c$. Suppose $\sup _{s, u}\left|g\left(\left[2^{n} s\right] / 2^{n}, u\right)-g(s, u)\right|<\varepsilon$. Then

$$
\begin{aligned}
& \left|\int_{0}^{t} \int_{\Gamma} g(s, u) \lambda_{k}(d s, d u)-\int_{0}^{t} \int_{\Gamma} g(s, u) \lambda(d s, d u)\right| \\
& \quad \leq \int_{0}^{t} \int_{\Gamma}\left|g(s, u)-g\left(\frac{\left[2^{n} s\right]}{2^{n}}, u\right)\right|\left(\lambda_{k}(d s, d u)+\lambda(d s, d u)\right) \\
& \quad+\left|\int_{0}^{t} \int_{\Gamma} g\left(\frac{\left[2^{n} s\right]}{2^{n}}, u\right) \lambda_{k}(d s, d u)-\int_{0}^{t} \int_{\Gamma} g\left(\frac{\left[2^{n} s\right]}{2^{n}}, u\right) \lambda(d s, d u)\right| \\
& \quad \leq 2 \varepsilon t+\text { 2nd term. }
\end{aligned}
$$

Appealing to (2.15), we see that the 2 nd term tends to 0 , as $k \rightarrow \infty$, for any $n$. Hence we can conclude (2.16).

Let $\zeta_{i}, i=1,2$, be $\tilde{M}_{T}$ (or $\hat{M}([0 T] \times \Gamma)$ )-valued random variables, which may be defined on different probability spaces. $\nu_{i}$ denotes the probability distribution of $\zeta_{i}$. So, $\nu_{i}$ is a probability on $\left(\tilde{M}_{T}, \tilde{d}_{T}\right)($ or $(\hat{M}([0 T] \times T)$, $\hat{d}_{T}$ ) respectively). Let $\tilde{m}_{T}$ (or $\hat{m}_{T}$ ) denote the totality of $\tilde{M}_{T}($ or $\hat{M}([0 T] \times \Gamma)$ )valued random variables. We endow the following Prohorov metric $\tilde{D}_{T}\left(\right.$ or $\hat{D}_{T}$ ) on $\tilde{m}_{T}$ (or $\hat{m}_{T}$ resp.),

$$
\begin{aligned}
& \tilde{D}_{T}\left(\zeta_{1}, \zeta_{2}\right)=L\left(\nu_{1}, \nu_{2}\right) \\
& \hat{D}_{T}\left(\zeta_{1}, \zeta_{2}\right)=L\left(\nu_{1}, \nu_{2}\right) .
\end{aligned}
$$

Since $\tilde{M}_{T}$ and $\hat{M}\left(\left[\begin{array}{ll}0 & T\end{array}\right] \times \Gamma\right)$ are compact metric spaces, $\left(\tilde{m}_{T}, \tilde{D}_{T}\right)$ and $\left(\hat{m}_{T}, \hat{D}_{T}\right)$ are also compact spaces.

For $\mathscr{A}=(\Omega, F, \stackrel{\circ}{P}, \xi, B, Y, q)$, we sometimes denote $\xi$ by $\xi_{\&}$ and so on, when any confusion might occur. Let $X\left(=X_{\mathscr{A}}\right)$ be a solution of $(2.1)$ for $\mathscr{A}$. Then $(X, \xi, B, Y, q)$ becomes a $M_{T}=C\left([0, T] \rightarrow R^{n}\right) \times R^{n} \times C\left([0 T] \rightarrow R^{n}\right)$ $\times\left(\left[\begin{array}{ll}0 & T\end{array}\right] \rightarrow R^{1}\right) \times \hat{M}\left(\left[\begin{array}{ll}0 & T\end{array}\right] \times \Gamma\right)$ valued random variable. Endowing $M_{T}$ 
with a usual metric $d_{T}$ (=sum of metric of each component) $M_{T}$ turns out as a complete separable metric space. Let $m_{T}$ denote the totality of $M_{T}$-valued random variables and $D_{T}$ the Prohorov metric on $m_{T}$. Hereafter we denote $(X, \xi, B, Y, q)$ by $(X, \mathscr{A})$ for simplicity if no confusion occurs. We also say that $\mathscr{A}_{n} \rightarrow \mathscr{A}$ (in Prohorov topology), if $\left(\xi_{n}, B_{n}, Y_{n}, q_{n}\right) \rightarrow$ $(\xi, B, Y, q)$ in Prohorov topology. $\Omega_{n}, F_{n}, P_{n}$ can also depend on $n$. $\xi_{\star}$, $B_{\mathscr{A}}$ and $\left(Y_{\mathscr{A}}, q_{\mathscr{A}}\right)$ are independent for any $\mathscr{A} \in \mathfrak{A}$ and $B_{\mathscr{A}}$ is a Brownian motion. Therefore we have

Proposition 2.2. $\mathscr{A}_{n} \rightarrow \mathscr{A}$, iff $\xi_{n} \rightarrow \xi$ in law and $\left(Y_{n}, q_{n}\right) \rightarrow(Y, q)$ in Prohorov topology.

Now we put the set $\mathscr{P}_{\mu}=$ totality of probability distributions of $\left(Y_{\mathscr{A}}, q_{\mathscr{\alpha}}\right)$, $\mathscr{A} \in \mathfrak{A}(\mu), \mathfrak{Y}(\mu)$ defined later (3.8). Since $\xi_{\mathscr{A}}, B_{\mathscr{A}}$ and $\left(Y_{\mathscr{A}}, q_{\mathscr{A}}\right)$ are independent for $\mathscr{A} \in \mathfrak{R}, \mathscr{P}_{\mu}$ does not depend on $\mu$, say $\mathscr{P}$. Moreover $\pi \in \mathscr{P}$, iff $\pi$ is a probability on $C\left(\left[\begin{array}{ll}0 & T\end{array}\right] \rightarrow R^{1}\right) \times \hat{M}\left(\left[\begin{array}{ll}0 & T\end{array}\right] \times \Gamma\right)$ such that the first component $y$ is a Brownian motion under $\pi$ and its increments $y(t)-y(s)$ is independent of $\sigma_{s}(y, \lambda)$ for $t>s$, where $\lambda$ is the second component, (see $\S 2$ of Fleming-Pardoux [8]). Since $C\left(\left[\begin{array}{ll}0 & T\end{array}\right] \rightarrow R^{1}\right) \times \hat{M}\left(\left[\begin{array}{ll}0 & T\end{array}\right] \times \Gamma\right)$ is a product of complete separable metric space, it becomes a complete separable metric space. So we introduce the Prohorov topology on $\mathscr{P}$. Then $\mathscr{P}$ is a compact metric space, because the first component is a Browian motion and $\hat{M}\left(\left[\begin{array}{ll}0 & T\end{array}\right] \times \Gamma\right)$ is a compact metric space. Now we have

Proposition 2.3.

i) $\mathscr{P}=$ totality of probability distribution of $\left(Y_{\mathscr{A}}, q_{\mathscr{A}}\right), \mathscr{A} \in \mathfrak{A}$, is a compact metric space with Prohorov metric.

ii) $\mathscr{P}=$ totality of probability distribution of $\left(Y_{\mathscr{A}}, q_{\mathscr{A}}\right), \mathscr{A} \in \mathfrak{A}(\mu)$, for any $\mu$.

iii) For $\mathscr{A}, \mathscr{A}^{\prime} \in \mathfrak{H}(\mu), D_{T}\left(\left(X_{\mathscr{A}}, \mathscr{A}\right),\left(X_{\mathscr{A}^{\prime}}, \mathscr{A}^{\prime}\right)\right)=0$ iff probability distri-

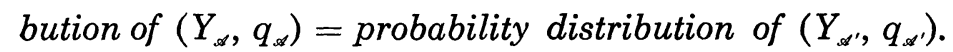

\section{§ 3. Existence of optimal relaxed control}

Let $N$ be a compact subset of probability measures on $R^{n}$ with Prohorov metric. Put $P_{\eta}=$ Probability law of $\eta$ and

$$
\mathfrak{R}=\left\{(X, \mathscr{A}) ; P_{\xi_{\infty}} \in N\right\} .
$$

Proposition 3.1. $\mathfrak{R}$ is a compact subset of $\left(m_{T}, D_{T}\right)$. 
Proof. By the condition (A1), $\left\{X_{s} ; P_{\xi_{s}} \in N\right\}$ is totally bounded in Prohorov topology. $B_{s}$ and $Y_{s}$ are Brownian motions for any $\mathscr{A} \in \mathfrak{A}$. Since $\hat{m}_{T}$ is compact, $\left\{q_{\star} ; \mathscr{A} \in \mathfrak{A}\right\}$ is totality bounded. Therefore

$$
\Re=\left\{(X, \mathscr{A}) ; P_{\xi_{\mathscr{S}}} \in N\right\}
$$

is a totally bounded subset of $\left(m_{T}, D_{T}\right)$.

Now we will show that $\mathfrak{N}$ is closed. Let $\left(X_{k}, \mathscr{A}_{k}\right), k=1,2, \cdots$ be a Cauchy sequence. Using Skorobod's theorem, we can construct $\left(X_{k}^{*}, \xi_{k}^{*}\right.$, $\left.B_{k}^{*}, Y_{k}^{*}, q_{*}^{*}\right)$ and $\left(X^{*}, \xi^{*}, B^{*}, Y^{*}, q^{*}\right)$ on a probability space $\left(\Omega^{*}, F^{*}, \stackrel{P}{ }^{*}\right)$, so that

(3.1) $\left(X_{k}^{*}, \xi_{k}^{*}, B_{k}^{*}, Y_{k}^{*}, q_{k}^{*}\right)$ has the same probability law as $\left(X_{k}, \xi_{k}, B_{k}, Y_{k}\right.$, $\left.q_{k}\right), k=1,2, \cdots$.

(3.2) As $k \rightarrow \infty,\left(X_{k}^{*}, \xi_{k}^{*}, B_{k}^{*}, Y_{k}^{*}, q_{k}^{*}\right)$ converges to $\left(X^{*}, \xi^{*}, B^{*}, Y^{*}, q^{*}\right)$ in $d_{T}$ metric, $\stackrel{\circ}{*}^{*}$-almost surely.

Hence $\xi^{*}, B^{*}$ and $\left(Y^{*}, q^{*}\right)$ are independent and $B^{*}$ and $Y^{*}$ are Brownian motions. Moreover we see that, for a.a. $\omega\left(\dot{P}^{*}\right), q^{*}(\cdot, \omega) \in \hat{M}\left(\left[\begin{array}{ll}0 & T\end{array}\right] \times \Gamma\right)$ and $\hat{d}_{T}\left(q_{k}^{*}(\cdot, \omega), q^{*}(\cdot, \omega)\right)$ tends to 0 as $k \rightarrow \infty$, by virtue of Proposition 2.2. On the other hand (2.15) implies that $q^{*}(t, A, \cdot)$ is $F^{*}$-measurable. Since $q(t, A, \omega)$ is continuous in $t, q^{*}(\cdot, A, \cdot)$ is $B_{1}[0 T] \times F^{*}$-measurable. Namely $q^{*}$ satisfies the conditions (v) and (vi). (3.2) again tells us that $Y^{*}(t)$ $-Y^{*}\left(r_{i}\right)$ is independent of $\sigma_{s}\left(Y^{*}, q^{*}\right)$ whenever $s \leq r_{i} \leq t$. Since $Y^{*}$ has continuous paths, this implies that $Y^{*}(t)-Y^{*}(s)$ is independent of $\sigma_{s}\left(Y^{*}\right.$, $\left.q^{*}\right)$, Therefore $\mathscr{A}^{*}=\left(\Omega^{*}, F^{*}, \stackrel{\circ}{ }^{*}, \xi^{*}, B^{*}, Y^{*}, q^{*}\right) \in \mathfrak{A}$.

Next we will show that $X^{*}$ is a solution of (2.1) for $\mathscr{A}^{*}$.

$$
\begin{aligned}
& \left|\int_{0}^{t} \int_{\Gamma} \gamma\left(X_{k}^{*}(s, \omega), u\right) q_{k}^{*}(d s, d u, \omega)-\int_{0}^{t} \int_{\Gamma} \gamma\left(X^{*}(s, \omega), u\right) q^{*}(d s, d u, \omega)\right| \\
& \quad \leq \int_{0}^{t} \int_{\Gamma}\left|\gamma\left(X_{k}^{*}(s, \omega), u\right)-\gamma\left(X^{*}(s, \omega), u\right)\right| q_{k}^{*}(d s, d u, \omega) \\
& \quad+\mid \int_{0}^{t} \int_{\Gamma} \gamma\left(X^{*}(s, \omega), u\right)\left(q_{k}^{*}(d s, d u, \omega)-q^{*}(d s, d u, \omega)\right)
\end{aligned}
$$

the 1st term $\quad \leq K \int_{0}^{t}\left|X_{k}^{*}(s, \omega)-X^{*}(s, \omega)\right| q_{k}^{*}(d s, \Gamma, \omega)$

$$
=K \int_{0}^{t}\left|X_{k}^{*}(s, \omega)-X^{*}(s, \omega)\right| d s .
$$

Since $X_{k}^{*}(\cdot, \omega)$ converges to $X^{*}(\cdot, \omega)$ uniformly in $[0, T]$, the 1st term 
converges to 0 , as $k \rightarrow \infty$. Appealing to Remark of Proposition 2.1, the 2nd term converges to 0 , as $k \rightarrow \infty$. So, we have

$$
\int_{0}^{t} \int_{\Gamma} \gamma\left(X_{k}^{*}(s, \omega), u\right) q_{k}^{*}(d s, d u, \omega) \longrightarrow \int_{0}^{t} \int_{\Gamma} \gamma\left(X^{*}(s, \omega) q^{*}(d s, d u, \omega) .\right.
$$

Using a routine method we get

$$
\int_{0}^{t} \alpha\left(X_{k}^{*}(s) d B_{k}^{*}(s)\right) \longrightarrow \int_{0}^{t} \alpha\left(X^{*}(s) d B^{*}(s)\right) \quad \text { in proba }\left(\stackrel{P}{ }^{*}\right)
$$

From (3.4) and (3.5), we conclude that $X^{*}$ is a solution of (2.1) for $\mathscr{A}^{*}$. This completes the proof of Proposition 3.1 .

Corollary. If $\mathscr{A}_{k} \rightarrow \mathscr{A}$, then $\left(X_{k}, \mathscr{A}_{k}\right) \rightarrow(X, \mathscr{A})$ in $D_{T}$.

Let $f$ and $h$ be bounded and uniformly continuous functions on $R^{n}$. Define a pay-off function $J(\mathscr{A})$ as follows,

$$
J(\mathscr{A})=\stackrel{\circ}{E} f\left(X_{\mathscr{A}}(T)\right) L(T, \mathscr{A})
$$

where $\stackrel{\circ}{E}$ stands for the expectation in $(\Omega, F, \stackrel{\circ}{P})$, and

$$
L(T, \mathscr{A})=\exp \left(\int_{0}^{T} h(X(s)) d Y(s)-\frac{1}{2} \int_{0}^{T}|h(X(s))|^{2} d s\right)
$$

where $X=X_{\star}$ and $Y=Y_{\star}$.

For a probability measure $\mu$, we denote

$$
\mathfrak{X}(\mu)=\left\{\mathscr{A} \in \mathfrak{X} ; P_{\xi_{\mathscr{A}}}=\mu\right\}
$$

i.e. the set of all admissible system where initial distribution equals to $\mu$.

For a given $\mu$ we want to maximize $J(\mathscr{A})$ by a suitable choice of $\mathscr{A} \in \mathfrak{A}(\mu)$.

TheOREm 2. There exists an optimal admissible system $\tilde{A} \in \mathfrak{A}(\mu)$, that is

$$
\sup _{\mathscr{A} \in \mathscr{R}(\mu)} J(\mathscr{A})=J(\tilde{\mathscr{A}}) .
$$

Proof. Let $\mathscr{A}_{k} \in \mathfrak{U}(\mu)$ be approximately optimal, i.e.

$$
\lim _{k \rightarrow \infty} J\left(\mathscr{A}_{k}\right)=\sup _{\mathscr{A} \in \mathscr{R}(\mu)} J(\mathscr{A}) .
$$

By virtue of Proposition 3.1, some subsequence $\left(X_{k_{i}}, \mathscr{A}_{k_{i}}\right)$ converges to $(X, \mathscr{A})$ in Prohorov topology. For simplicity we may assume $\left(X_{k}, \mathscr{A}_{k}\right) \rightarrow$ 
$(X, \mathscr{A})$ as $k \rightarrow \infty$. Again Skorobod's theorem tells us that their suitable version satisfy (3.1) and (3.2). So we again assume that $\left(X_{k}, \mathscr{A}_{k}\right)$ and $(X, \mathscr{A})$ satisfy (3.1) and (3.2), since $J(\mathscr{A})$ depends on only probability law. From boundedness of $f$ and $h$, we have

$$
\stackrel{\circ}{E}\left(f\left(X_{k}(T)\right) L\left(T, \mathscr{A}_{k}\right)\right)^{2} \leq\|f\|^{2} e^{2 T\|h\|}, \quad k=1,2, \cdots .
$$

Hence $\left\{f\left(X_{k}(T)\right) L\left(T, \mathscr{A}_{k}\right), k=1,2, \cdots\right\}$ is uniformly integrable. On the other hand $L\left(T, \mathscr{A}_{k}\right)$ tends to $L(T, \mathscr{A})$ in proba. Appealing to the convergence theorem we get

$$
\lim _{k \rightarrow \infty} J\left(\mathscr{A}_{k}\right)=J(\mathscr{A}) .
$$

Combining (3.12) with (3.10), we complete the proof.

Remark. Appealing to Corollary of Proposition 3.1, we see that if $\mathscr{A}_{k} \rightarrow \mathscr{A}$, then $J\left(\mathscr{A}_{k}\right) \rightarrow J(\mathscr{A})$.

Now we treat the following case; $r(x, u)=b_{1}(x)+b_{2}(x) u$ where $b_{2}(x)$ is $n \times k$ matrix.

THEOREM 3. If $r(x, u)=b_{1}(x)+b_{2}(x) u$, then $q=q_{s}$ can be replaced by a $\Gamma$-valued $\sigma_{t}(q)$-progressively measurable process $U$ (i.e. usual admissible control under partial observation). That is, $X=X_{s}$ is a unique solution of the following S.D.E.

$$
\left\{\begin{array}{l}
d X(t)=\alpha(X(t)) d B(t)+\gamma(X(t), U(t)) d t \\
X(0)=\xi
\end{array}\right.
$$

where $B=B_{s}$ and $\xi=\xi_{s}$.

Proof. Our required $U$ is obtained by the following lemma.

Lemma. There exists a $\sigma_{t}(q)$-progressively measurable $\Gamma$-valued process $U$ such that

$$
\int_{0}^{t} \int_{\Gamma} u q(d s, d u)=\int_{0}^{t} U(s) d s, \quad \text { for } \forall_{t} \leq T .
$$

Proof. Define $U_{k}$ as follows

$$
U_{k}(t, \omega)=\left\{\begin{array}{l}
2^{k} \int_{\Gamma} u\left(q(t, d u, \omega)-q\left(t-2^{-k}, d u, \omega\right)\right), \quad \text { for } t \geq 2^{-k} \\
\frac{1}{t} \int_{\Gamma} u q(t, d u, \omega), \quad \text { for } 0<t<2^{-k}
\end{array}\right.
$$


Since $\Gamma$ is convex compact, $U_{k}(t, \omega) \in \Gamma$ and $\sigma_{t}(q)$-progressively measurable. Moreover the compactness of $\Gamma$ tells us that $\left\{U_{k}, k=1,2, \cdots\right\}$ is weakly totally bounded in $L^{2}\left(\left[\begin{array}{ll}0 & T\end{array}\right] \times \Omega\right)$. Hence some subsequence converges weakly and their suitable convex combinations converge strongly; say $\sum_{p=\ell}^{N \ell} \sigma_{p}^{\ell} U_{n_{p}}(t, \omega)$ converges to $U(t, \omega)$ in $L^{2}\left(\left[\begin{array}{ll}0 & T\end{array}\right] \times \Omega\right)$, as $\ell \rightarrow \infty$. So $U$ is a $\Gamma$-valued $\sigma_{t}(q)$-progressively measurable process.

On the other hand the definition of $U_{k}$ implies

$$
\int_{0}^{t} U_{k}(s, \omega) d s \rightarrow \int_{\Gamma} u q(t, d u, \omega)\left(=\int_{0}^{t} \int_{\Gamma} u q(d s, d u, \omega)\right) .
$$

as $k \rightarrow \infty$. Taking the convex combination of $U_{n_{p}}$, we can conclude that $U$ satisfies (3.14) by bounded convergence theorem.

Now we return to the proof of Theorem 3. Since $\xi, B$ and $U$ are independent, (3.13) has a unique solution. So it is enough to show that $X=X_{\star}$ satisfies (3.13). By the Lemma we can see

$$
\int_{0}^{t} b_{2}(X(s)) \int_{\Gamma} u q(d s, d u)=\int_{0}^{t} b_{2}(X(s)) U(s) d s .
$$

Using " $\int_{0}^{t} b_{1}(X(s)) q(d s, d u)=\int_{0}^{t} b_{1}(X(s)) d s "$ ", we have

$$
\int_{0}^{t} \int_{\Gamma} \gamma(X(s), u) q(d s, d u)=\int_{0}^{t} \gamma(X(s), U(s)) d s .
$$

This completes the proof of Theorem 3 .

Definition 3. A $\Gamma$-valued process $U$ is called an admissible control under partial observation, if $\xi, B$ and $(Y, U)$ are independent and $Y(t)-$ $Y(s)$ is independent of $\sigma_{s}(Y, U)$. Precisely speaking $\mathscr{A}_{U}=(\Omega, F, \stackrel{\AA}{P}, \xi, B$, $Y, U)$ is called an admissible usual system.

An admissible control $U$ can be regarded as the following relaxed control $q$,

$$
q(t, A, \omega)=\int_{0}^{t} \delta_{U(s, \omega)}(A) d s=|s \leq t ; U(s, \omega) \in A|
$$

where $\delta_{a}$ is the $\delta$-measure at $a$. Appealing to Theorems 2 and 3 , we can derived,

CoRollary. If $r(x, u)=b_{1}(x)+b_{2}(x) u$, then there exists an optimal admissible usual system $\tilde{\mathscr{A}}_{U}$. That is,

$$
J\left(\tilde{\mathscr{A}}_{U}\right)=\sup _{\mathscr{A} U: \text { ad. usual sys }} J\left(\mathscr{A}_{U}\right)=\sup _{\mathscr{A} \in \mathscr{\mathscr { A }}(u)} J(\mathscr{A}) .
$$


This fact was directly proved by Haussmann [9] and in a slightly different form by Fleming-Pardoux [8].

\section{§4. Approximation by usual controls}

For $\mathscr{A}=(\Omega, F, \stackrel{P}{P}, \xi, B, Y, q)$ we define $P_{n}$ by

$$
P_{n}(t, A, \omega)=\left\{\begin{array}{l}
\frac{1}{t} q(t, A, \omega) \quad 0<t<2^{-n} \\
\left(q(t, A, \omega)-q\left(t-2^{-n}, A, \omega\right)\right) 2^{n}, \quad 2^{-n} \leq t,
\end{array}\right.
$$

namely $P_{n}$ is an approximate time derivative of $q . P_{n}(\cdot, A, \cdot)$ is $\sigma_{t}(q)$ progressively measurable and $P_{n}(t, \cdot, \omega)$ is a probability on $\Gamma$. Define $q_{n}$ by

$$
q_{n}(t, A, \omega)=\int_{0}^{t} P_{n}(s, A, \omega) d s .
$$

Then $q_{n}$ satisfies the conditions (v) and (vi) and $\mathscr{A}_{n}=\left(\Omega, F, \stackrel{P}{P}, \xi, B, Y, q_{n}\right)$ $\in \mathfrak{A}$. Since we have

$$
\begin{array}{r}
\left|q_{n}(t, A, \omega)-q(t, A, \omega)\right| \leq 2^{-n}+2^{n} \int_{t-2^{-n}}^{t}|q(s, A, \omega)-q(t, A, \omega)| d s, \\
\text { for a.a. } \omega(\stackrel{P}{P}),
\end{array}
$$

the condition (iv) implies, as $n \rightarrow \infty$,

$$
\sup _{A}\left|q_{n}(t, A, \omega)-q(t, A, \omega)\right| \rightarrow 0, \quad \text { uniformly on }[0, T] .
$$

and

$$
\hat{d}_{T}\left(q_{n}(\cdot, \omega), q(\cdot, \omega)\right) \longrightarrow 0 .
$$

Fix $u_{0} \in \Gamma$ arbitrarily and define $P_{n, k}$ by

$$
P_{n, k}(t, A, \omega)= \begin{cases}P_{n}\left(\frac{\left[2^{k} t\right]}{2^{k}}, A, \omega\right), & \text { for } t \geq 2^{-k} \\ \delta_{u_{0}}(A) & \text { for } t<2^{-k}\end{cases}
$$

Then $P_{n, k}$ is a step function in the time variable $t$. Put $q_{n, k}$ as follows.

$$
q_{n, k}(t, A, \omega)=\int_{0}^{t} P_{n, k}(s, A, \omega) d s .
$$

We call $q_{n, k}$ a switching relaxed control with interval $2^{-k}$. It is clear that $\left(\Omega, F, \stackrel{\circ}{P}, \xi, B, Y, q_{n, k}\right) \in \mathfrak{A}$ and

$$
\left|q_{n, k}(t, A, \omega)-q_{n}(t, A, \omega)\right| \leq 2^{n-k-1} t+2^{-k} .
$$


Therefore we get

$$
\lim _{k \rightarrow \infty} \sup _{t \leq T, A}\left|q_{n, k}(t, A, \omega)-q_{n}(t, A, \omega)\right|=0 .
$$

Now we conclude the following proposition,

Proposition 4.1. For $\mathscr{A}=(\Omega, F, \stackrel{\circ}{P}, \xi, B, Y, q) \in \mathfrak{U}$, there exists an approximate sequence of switching relaxed control $q_{k}$ with interval $2^{-k}$, such that $q_{k}(\cdot, A, \cdot)$ is $\sigma_{t}(q)$-progressively measurable and moreover

$$
\lim _{k \rightarrow \infty} \sup _{0 \leq t \leq T, A}\left|q_{k}(t, A)-q(t, A)\right|=0, \quad \stackrel{\circ}{P} \text {-almost surely }
$$

and

$$
\lim _{k \rightarrow \infty} \hat{d}_{T}\left(q_{k}, q\right)=0, \quad \stackrel{\circ}{\text { P-almost surely. }}
$$

Putting $\mathscr{A}_{k}=\left(\Omega, F, \stackrel{\circ}{P}, \xi, B, Y, q_{k}\right)$, we can see the following corollary, by virtue of the Remark of Theorem 2 .

CoROLlaRY. There exists an approximate admissible switching system $\mathscr{A}_{k}$, such that

$$
\mathscr{A}_{k} \longrightarrow \mathscr{A}, \quad \text { as } k \rightarrow \infty \text {. }
$$

Hence $J\left(\mathscr{A}_{k}\right)$ converges to $J(\mathscr{A})$.

TheOREM 4. There exists a $\Gamma$-valued $\sigma_{t}(q)$-progressively measurable process $U_{k}$, such that

$$
q_{k}(t, A, \omega)=\int_{0}^{t} \delta_{U_{k}(s, \omega)}(A) d s
$$

approximates $q$ in the following sence; $\mathscr{A}_{k}=\left(\Omega, F, \stackrel{\circ}{P}, \xi, B, Y, q_{k}\right)$ satisfies (4.12).

Proof. By the Corollary of Proposition 4.1, we may assume that $\mathscr{A}$ is an admissible switching system with interval $2^{-N}$. Appealing to a Chattering Lemma [5], we will construct our desired $U_{k_{i}}$ in the following way.

Let $\left\{u_{1}, \cdots, u_{m}\right\}$ be an $\varepsilon$-net of $\Gamma$, and $V_{1}, \cdots, V_{m} \in \boldsymbol{B}(\Gamma)$ a partition of $\Gamma$ such that

$$
\left|u_{i}-u\right|<\varepsilon \quad \text { for } \forall u \in V_{i} \text {. }
$$

Since a given $q$ is a switching relaxed control, it can be written by

$$
q(t, A, \omega)=\int_{0}^{t} p(s, A, \omega) d s
$$


with $p$ of step function in $s$. Define $\hat{p}=\hat{p}_{\varepsilon}$ and $\hat{q}=\hat{q}_{\mathrm{s}}$ as follows,

$$
\hat{p}\left(s,\left\{u_{i}\right\}, \omega\right)=p\left(s, V_{i}, \omega\right)
$$

and

$$
\hat{q}\left(t,\left\{u_{i}\right\}, \omega\right)=\int_{0}^{t} \hat{p}\left(s,\left\{u_{i}\right\}, \omega\right) d s .
$$

Then $\hat{p}(t, \cdot, \omega)$ is a discrete probability on $\Gamma$ and for $\forall g \in C(\Gamma)$

$$
\int_{\Gamma} g(u) \hat{p}_{\varepsilon}(t, d u, \omega) \longrightarrow \int_{\Gamma} g(u) p(t, d u, \omega), \quad \text { as } \quad \varepsilon \downarrow 0 .
$$

Define $\theta_{i}, i=0, \cdots, m$ as follows: Let $j^{2-N} \leq s_{1}<s_{2}<(j+1) 2^{-N}$,

$$
\begin{aligned}
& \theta_{0}(\omega)=s_{1} \\
& \theta_{i}(\omega)=\sum_{\ell=1}^{i} \int_{s_{1}}^{s_{2}} \hat{p}\left(t,\left\{u_{\ell}\right\}, \omega\right) d t+s_{1}, \quad i=1, \cdots, m .
\end{aligned}
$$

Then $s_{1}=\theta_{0}(\omega) \leq \theta_{1}(\omega) \leq \cdots \leq \theta_{m}(\omega)=s_{2}$ and

$$
\begin{gathered}
\int_{s_{1}}^{s_{2}} \int_{\Gamma} g(u) \hat{q}(d t, d u, \omega)=\int_{s_{1}}^{s_{2}} \int_{\Gamma} g(u) \hat{p}(t, d u, \omega) d t \\
=\sum_{i=1}^{m} g\left(u_{i}\right) \int_{s_{1}}^{s_{2}} \hat{p}\left(t,\left\{u_{i}\right\}, \omega\right) d t=\sum_{i=1}^{m} g\left(u_{i}\right)\left(\theta_{i}(\omega)-\theta_{i-1}(\omega)\right) \\
=\int_{s_{1}}^{s_{2}} g(U(t, \omega)) d t=\int_{s_{1}}^{s_{2}} \int_{\Gamma} g(u) \delta_{U(t, \omega)}(d u) d t
\end{gathered}
$$

where

$$
U(t, \omega)=U_{\varepsilon, s_{1}, s_{2}}(t, \omega)=u_{i} \quad \text { on }\left[\left(\theta_{i-1}(\omega), \theta_{i}(\omega)\right) .\right.
$$

Therefore $U_{\varepsilon, s_{1}, s_{2}}(t)$ is $\sigma_{j 2-N}(q)$-measurable. Putting $\varepsilon=2^{-k}, s_{1}=\ell 2^{-k}(k>N)$, we define $U_{k}$ by

$$
\begin{aligned}
& U_{k}(t, \omega)=U_{2^{-k}, \ell 2^{-k},(\ell+1) 2^{-k}}(t, \omega) \\
& \quad \text { for } \ell 2^{-k} \leq t<(\ell+1) 2^{-k}, \ell=0,1,2 \cdots .
\end{aligned}
$$

Consider the SDE

$$
\left\{\begin{array}{l}
d \xi_{k}(t)=\alpha\left(\xi_{k}(t)\right) d B(t)+\gamma\left(\xi_{k}(t), U_{k}(t)\right) d t \\
\xi_{k}(0)=\xi .
\end{array}\right.
$$

If we regard $U_{k}(t)$ as $\delta_{U_{k}(t)}$, then $\xi_{k}$ turns out a solution of (2.1) for $\mathscr{A}_{k}=$ $\left(\Omega, F, \stackrel{\circ}{P}, \xi, B, Y, q_{k}\right)$ where $q_{k}(t, A, \omega)=\int_{0}^{t} \delta_{U_{k}(s, \omega)}(A) d s$. Moreover (4.19) means 


$$
q_{k}\left(\ell 2^{-k}, \cdot, \omega\right)=\hat{q}_{2-k}\left(\ell 2^{-k}, \cdot, \omega\right), \quad \ell=0,1, \cdots,\left[2^{\ell} T\right] .
$$

Hence, combining with (4.17), we can see that, as $k \rightarrow \infty$,

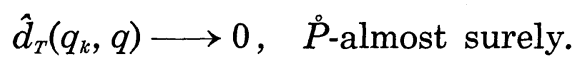

Evidently this completes the proof.

\section{§5. Continuity of conditional expectation}

According to [8] we define $L$ pathwise. Hereafter we assume the following smoothness on $h$.

(A3) $h, \frac{\partial h}{\partial x_{i}}, \frac{\partial^{2} h}{\partial x_{i} \partial x_{j}} i, j=1, \cdots, n$ are bounded and uniformly continuous.

Putting $X=X_{\mathscr{s}}$, Ito's formula tells us that

$$
\begin{gathered}
\int_{0}^{T} h(X(t)) d Y(t)=h(X(T)) Y(T)-\int_{0}^{T} Y(t) d h(X(t)) \\
=h(X(T)) Y(T)-\sum_{i j} \int_{0}^{T} Y(t) \frac{\partial h}{\partial x_{i}}(X(t)) \alpha_{i j}(X(t)) d B_{j} \\
\int_{0}^{T} Y(t) A(t, q) h(X(t)) d t
\end{gathered}
$$

where

$$
A(t, q) h=\frac{1}{2} \sum a_{i j}(x) \frac{\partial^{2} h}{\partial x_{i} \partial x_{j}}+\sum R_{i}(t, x, \omega ; q) \frac{\partial h}{\partial x_{i}}
$$

with

$$
R(t, x, \omega ; q)=\varlimsup_{n \rightarrow \infty} 2^{n} \int_{\Gamma} \gamma(x, u)\left(q(t, d u, \omega)-q\left(t-2^{-n}, d u, \omega\right)\right)
$$

So, $R$ is $\sigma_{t}(q)$-progressively measurable for any $x$, and Lipschitz continuous with respect to $x$. Moreover for any $(x, \omega)$,

$$
R(t, x, \omega ; q)=\frac{\partial}{\partial t} \int_{\Gamma} \gamma(x, u) q(t, d u, \omega) \quad \text { for a.a. } t .
$$

For $y \in C\left(\left[\begin{array}{ll}0 & T\end{array}\right] \rightarrow R^{n}\right)$ and $\lambda \in \hat{M}\left(\left[\begin{array}{ll}0 & T\end{array}\right] \times \Gamma\right)$ we define $\mathscr{L}$ by

$$
\begin{aligned}
& \mathscr{L}(\theta, \xi, B, y, \lambda) \\
& =\exp \left[y(\theta) h(\eta(\theta))-\int_{0}^{\theta} y(s) A(s, \lambda) h(\eta(s)) d s-\frac{1}{2} \int_{0}^{\theta}|h(\eta(s))|^{2} d s\right. \\
& \left.\quad-\sum_{i j} \int_{0}^{s} y(s) \frac{\partial h}{\partial x_{i}}(\eta(s)) \alpha_{i j}(\eta(s)) d B_{j}(s)\right]
\end{aligned}
$$


where $\eta$ is a solution of S.D.E.

$$
\left\{\begin{array}{l}
d \eta(t)=\alpha(\eta(t)) d B(t)+\int_{\Gamma} \gamma(y(t), u) \lambda(d t, d u) \\
\eta(0)=\xi
\end{array}\right.
$$

Applying a successive approximation, we can see that $\eta$ is a Borel function of $\xi, B$ and $\lambda$. Hence $\mathscr{L}$ is Borel measurable with respect to $\theta, \xi, B$, $y, \lambda$. From (A1) and (A3) we have the following evaluation,

$$
\begin{gathered}
e^{-F(y, \theta)} \exp \left[-\sum_{i j} \int_{0}^{\theta} y(t) \frac{\partial h}{\partial x_{i}}(\eta(t)) \alpha_{i j}(\eta(t)) d B_{j}(t)\right. \\
\left.-\frac{1}{2} \int_{0}^{t} \sum_{j}\left(y(t) \sum_{i} \frac{\partial h}{\partial x_{i}}(\eta(t)) \alpha_{i j}(\eta(t))\right)^{2} d t\right] \\
\leq \mathscr{L}(\theta, \xi, B, y, \lambda) \leq e^{F(y, \theta)} \exp \left[-\sum_{i j} \int_{0}^{\theta} \cdots d B_{j}(t)-\frac{1}{2} \int_{0}^{t} \cdots d t\right]
\end{gathered}
$$

where

$$
F(y, \theta)=K_{1}\left(\sup _{t \leq \theta}|y(t)|+1\right)^{2}(\theta+1) .
$$

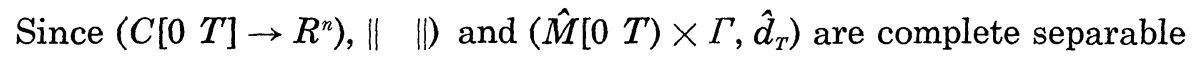
metric spaces, the regular conditional probablity $\stackrel{\circ}{P}((X, \xi, B, Y, q) \in \cdot / Y=y$, $q=\lambda$ ) exists. This regular conditional probability is nothing but the probability distribution of $(\eta, \xi, B, y, \lambda)$, because $(\xi, B)$ and $(Y, q)$ are independent. Putting $\mu=P_{\xi}(=$ probability distribution of $\xi$ ), we have a version of conditional expectation as follows.

$$
\begin{aligned}
& \stackrel{\circ}{E}(f(X(\theta)) L(\theta, \mathscr{A}) / Y=y, q=\lambda)=\stackrel{\circ}{E} f(\eta(\theta)) \mathscr{L}(\theta, \xi, B, y, \lambda) \\
& \quad=\int \stackrel{\circ}{E} f(\eta(\theta, x)) \mathscr{L}(\theta, x, B, y, \lambda) d \mu(x), \quad \text { for bounded Borel } f,
\end{aligned}
$$

where $\eta(\theta, x)$ is a solution of (5.6) with $\eta(0, x)=x$. The right side of (5.8) is Borel measurable with respect to $\theta, y, \lambda$, which depends on $f$ and $\mu$. So we denote the right side of (5.8) by $C(\theta, y, \lambda, \mu, f)$. Moreover $C(\theta, y$, $\lambda, \mu, f)$ depends on the value of $y$ and $\lambda$ up to time $\theta$. Stressing $\mu$ we denote $\stackrel{\circ}{E}$ by $\stackrel{\circ}{E}_{\mu}$. That is,

$$
\begin{aligned}
& C(\theta, Y, q, \mu, f)=\stackrel{\circ}{E}_{\mu}\left(f(X(\theta)) L(\theta, \mathscr{A}) / \sigma_{T}(Y, q)\right) \\
& \quad=\stackrel{\circ}{E}_{\mu}\left(f(X(\theta)) L(\theta, \mathscr{A}) / \sigma_{\theta}(Y, q)\right), \quad \stackrel{\circ}{P} \text {-almost surely. }
\end{aligned}
$$

Using (5.7) we have

$$
e^{-F(Y, \theta)} \leq C(\theta, Y, q, \mu, 1) \leq e^{F(Y, \theta)} .
$$

Now we define $C(\theta, Y, q, \nu, f)$ for a positive measure $\nu$ as follows 


$$
C(\theta, Y, q, \nu, f)=\|\nu\| C\left(\theta, Y, q, \frac{\nu}{\|\nu\|}, f\right)
$$

where $\|\nu\|=\nu\left(R^{n}\right)$ and we apply the same notations $\dot{E}_{\nu}$ for a general positive measure $\nu$, that is

$$
\stackrel{\circ}{E}_{\nu}\left(f(X(\theta)) L(\theta, \mathscr{A}) / \sigma_{\theta}(Y, q)\right)=C(\theta, Y, q, \nu, f)
$$

with $\mathscr{A}=(\Omega, F, \stackrel{\circ}{P}, \xi, B, Y, q)$ for $P_{\xi}=\nu /\|\nu\|$. The left side of (5.12) stands for

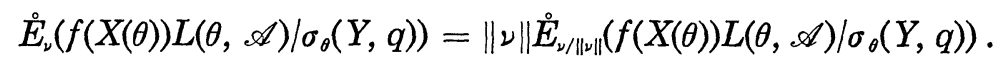

Since $\xi$ is independent of $(B, Y, q)$, the right side of (5.13) does not depend on a special choice of $\xi$.

Define $\Lambda(\theta, y, \lambda, \nu)(A)$ by

$$
\Lambda(\theta, y, \lambda, \nu)(A)=C\left(\theta, y, \lambda, \nu, \chi_{A}\right), \quad A \in \boldsymbol{B}_{n} .
$$

Then $\Lambda(\theta, y, \lambda, \nu)$ is a positive measure on $R^{n}$ and for any bounded Borel function $f$.

$$
\langle f, \Lambda(\theta, y, \lambda, \nu)\rangle=C(\theta, y, \lambda, \nu, f)
$$

where $\langle f, \Lambda\rangle=\int_{R^{n}} f(x) \Lambda(d x)$. From (5.10) we see

$$
\|\nu\| e^{-F(y, \theta)} \leq\|\Lambda(\theta, y, \lambda, \nu)\| \leq\|\nu\| e^{F(y, \theta)} .
$$

On the other hand $\mathscr{L}(\theta, \xi, B, y, \lambda)$ is continuous in $\theta, \stackrel{\circ}{P}$-almost surely, and (5.7) implies the uniformly integrability of $\{\mathscr{L}(\theta, \xi, B, y, \lambda), \theta \in[0, T]\}$. Hence $\|\Lambda(\theta, y, \lambda, \nu)\|=\|\nu\| \stackrel{\circ}{E} \mathscr{L}(\theta, \xi, B, y, \lambda)$ is continuous in $\theta$.

Define a metric $\Delta$ on $M\left(R^{n}\right)$ (= totality of positive measure on $R^{n}$ ) as follows

$$
\Delta(\mu, \nu)=L\left(\frac{\mu}{\|\mu\|}, \frac{\nu}{\|\nu\|}\right)+|\|\mu\|-\|\nu\||+\left|\frac{1}{\|\mu\|}-\frac{1}{\|\nu\|}\right| .
$$

Then $\left(M\left(R^{n}\right), \Delta\right)$ is a complete separable metric space and $\nu_{k}, k=1,2, \cdots$ is a Cauchy sequence, iff $\left\langle f, \nu_{k}\right\rangle$ converges for any $f \in C_{b}\left(R^{n}\right)$, as $k \rightarrow \infty$. and $\lim _{k \rightarrow \infty}\left\langle 1, \nu_{k}\right\rangle\left(=\left\|\nu_{k}\right\|\right)>0$. Recalling Prohorov's theorem we have

Proposition 5.1. $N \supset M\left(R^{n}\right)$ is $\Delta$-totally bounded, iff there exist positive constants $c$ and $c^{\prime}$ and for $\forall_{\varepsilon}>0$ there is a compact subset $K_{\varepsilon} \subset R^{n}$ such that 


$$
c^{\prime} \leq\|\nu\| \leq c \text { and } \nu\left(K_{\varepsilon}^{c}\right)<\varepsilon \text { for } \nu \in N .
$$

Put $m=$ totality of $M\left(R^{n}\right)$-valued random variables, which may be defined on different probability spaces. We endow the Prohorov metric on $m$, (called $\delta$ metric), namely

$$
\delta\left(\zeta_{1}, \zeta_{2}\right)=L\left(\mu_{1}, \mu_{2}\right)
$$

where $\mu_{i}$ is the probability distribution of $\zeta_{i}$. Then $(m, \delta)$ is a complete separable metric space, because $\left(M\left(R^{n}\right), \Delta\right)$ is a complete separable metric space.

Concerning the continuity of $\Lambda$, we can prove the following theorem.

Theorem 5. If $y_{k} \rightarrow y$ in $C\left(\left[\begin{array}{ll}0 & T\end{array}\right] \rightarrow R^{1}\right), \nu_{k} \rightarrow \nu$ in $\Delta, \lambda_{k} \rightarrow \lambda$ in $\hat{d}_{T}$ and $\theta_{k} \rightarrow \theta$, then

$$
\Lambda\left(\theta_{k}, y_{k}, \lambda_{k}, \nu_{k}\right) \longrightarrow \Lambda(\theta, y, \lambda, \nu) \text { in } \Delta .
$$

Proof. Firstly we remark that

$$
\int_{0}^{s} R\left(t, x, \lambda_{k}\right) d t \longrightarrow \int_{0}^{s} R(t, x, \lambda) d t .
$$

Recalling the definition of $R$ for $\lambda \in \hat{M}\left(\left[\begin{array}{ll}0 & T\end{array}\right] \times \Gamma\right)$ (see (5.3)), we get, for any $x$,

$$
R\left(t, x, \lambda_{k}\right)=\lim _{\lambda \rightarrow \infty} \int_{\Gamma} \gamma(x, u)\left(\lambda_{k}(t, d u)-\lambda_{k}\left(t-2^{-n}, d u\right)\right) 2^{n}
$$

for a.a. $t$.

Hence the bounded convergence theorem implies

$$
\begin{aligned}
\int_{0}^{s} R\left(t, x, \lambda_{k}\right) d t= & \lim _{n \rightarrow \infty}\left[\int_{0}^{s} \int_{\Gamma} 2^{n} \gamma(x, u) \lambda_{k}(t, d u) d t\right. \\
& \left.\quad-\int_{2^{n}}^{s} \int_{\Gamma} 2^{n} \gamma(x, u) \lambda_{k}\left(t-2^{-n}, d u\right) d t\right] \\
= & \int_{\Gamma} \gamma(x, u) \lambda_{k}(s, d u) .
\end{aligned}
$$

Since $\lambda_{k} \rightarrow \lambda$ in $\hat{d}_{T}$, (5.22) means (5.20).

Consequently we can easily see

Lemma. If $\phi_{k} \rightarrow \phi$ and $\psi_{k} \rightarrow \psi$ in $C\left(\left[\begin{array}{ll}0 & T\end{array}\right] \rightarrow R^{n}\right)$ and $C\left(\left[\begin{array}{ll}0 & T\end{array}\right] \rightarrow R^{1}\right)$ respectively, then

$$
\int_{0}^{s} R\left(t, \phi_{k}(t), \lambda_{k}\right) \psi_{k}(t) d t \longrightarrow \int_{0}^{s} R(t, \phi(t), \lambda) \psi(t) d t
$$


Putting $\Lambda_{k}=\Lambda\left(\theta_{k}, y, \lambda_{k}, \nu_{k}\right)$ and $\Lambda=\Lambda(\theta, y, \lambda, \nu)$, we will show

$$
\left\langle f, \Lambda_{k}\right\rangle \longrightarrow\langle f, \Lambda\rangle \quad \text { for } f \in C_{b}\left(R^{n}\right) \text {. }
$$

Consider the SDE on $\left(\Omega_{k}, F_{k}, \stackrel{\circ}{P}_{k}^{\prime}\right.$

$$
\left\{\begin{array}{l}
d \eta_{k}(t)=\alpha\left(\eta_{k}(t)\right) d B_{k}(t)+\int_{\Gamma} r\left(\eta_{k}(t), u\right) \lambda_{k}(d t, d u) \\
\eta_{k}(0)=\xi_{k}
\end{array}\right.
$$

and on $(\Omega, F, \stackrel{\circ}{P})$

$$
\left\{\begin{array}{l}
d \eta(t)=\alpha(\eta(t)) d B(t)+\int_{\Gamma} \gamma(\eta(t), u) \lambda(d t, d u) \\
\eta(0)=\xi
\end{array}\right.
$$

where $\xi_{k}$ and $\xi$ have probability distributions $\nu_{k} /\left\|\nu_{k}\right\|$ and $\nu /\|\nu\|$ respectively. Since $\left\{\left(\eta_{k}, \xi_{k}, B_{k}\right), k=1,2, \cdots\right\}$ is totally bounded in Prohorov topology and any convergent subsequence tends to $(\eta, \xi, B)$ in Prohorov topology, $\left(\eta_{k}\right.$, $\left.\xi_{k}, B_{k}\right), k=1,2, \ldots$ itself converges to $(\eta, \xi, B)$ in Prohorov topology. Appealing to Skorobod's theorem, we will assume that $\Omega_{k}=\Omega, F_{k}=F$, $\stackrel{P}{P}_{k}=\stackrel{P}{P}$ and $\stackrel{P}{P}$-almost surely $\eta_{k} \rightarrow \eta$ in $C\left([0 T] \rightarrow R^{n}\right), B_{k} \rightarrow B$ in $C\left([0 T] \rightarrow R^{n}\right)$ and $\xi_{k} \rightarrow \xi$ in $R^{n}$. Therefore the lemma guarantees

$$
\int_{0}^{\theta_{k}} y_{k}(s) A\left(s, \lambda_{k}\right) h\left(\eta_{k}(s)\right) d s \longrightarrow \int_{0}^{\theta} y(s) A(s, \lambda) h(\eta(s)) d s,
$$

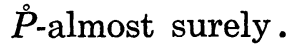

Furthermore, using a routine method we have

$$
\begin{aligned}
\int_{0}^{\theta_{k}} y_{k}(s) & \frac{\partial h}{\partial x_{i}}\left(\eta_{k}(s)\right) \alpha_{i j}\left(\eta_{k}(s)\right) d B_{k, .}(s) \\
& \longrightarrow \int_{0}^{\theta} y(s) \frac{\partial h}{\partial x_{i}}(\eta(s)) \alpha_{i j}(\gamma(s)) d B_{j}(s) \quad \text { in proba } \stackrel{P}{P}
\end{aligned}
$$

Hence we have

$$
\mathscr{L}\left(\theta_{k}, \xi_{k}, B_{k}, y_{k}, \lambda_{k}\right) \longrightarrow \mathscr{L}(\theta, \xi, B, y, \lambda) \quad \text { in proba } \stackrel{P}{P} .
$$

Since (5.7) means the uniformly integrability of $\left\{f\left(\eta_{k}\left(\theta_{k}\right) \mathscr{L}\left(\theta_{k} . \xi_{k}, B_{k}, y_{k}, \lambda_{k}\right)\right.\right.$, $k=1,2, \cdots\},(5.29)$ implies (5.24).

By virtue of Proposition 5.1, (5.16) and (5.20) guarantee the totally boundedness of $\left\{\Lambda_{k}, k=1,2, \cdots\right\}$. Consequently, again (5.24) tells us that $\Lambda_{k}$ converges to $A$ in metric $\Delta$. This completes the proof of Theorem 5 .

Now we apply this theorem to admissible systems. 
Theorem 6. Let $\mathscr{A}_{k}=\left(\Omega_{k}, F_{k}, \stackrel{\triangleright}{P}_{k}, \xi_{k}, B_{k}, Y_{k}, q_{k}\right)$ and $\mathscr{A}=(\Omega, F, \stackrel{P}{P}, \xi$, $B, Y, q)$ where $\xi_{k}$ and $\xi$ have probability distributions $\nu_{k} /\left\|\nu_{k}\right\|$ and $\nu /\|\nu\|$ respectively. If $\left(Y_{k}, q_{k}\right) \rightarrow(Y, q)$ in Prohorov topology, $\nu_{k} \rightarrow \nu$ in $\Delta$ and $\theta_{k}$ $\rightarrow \theta$, then

$$
\Lambda\left(\theta_{k}, Y_{k}, q_{k}, \nu_{k}\right) \longrightarrow \Lambda(\theta, Y, q, \nu) \quad \text { in metric } \delta \text {. }
$$

Proof. By the assumption $\mathscr{A}_{k} \rightarrow \mathscr{A}$ in Prohorov topology. Hence $\left(X_{k}, \mathscr{A}_{k}\right) \rightarrow(X, \mathscr{A})$ in Prohorov metric $D_{T}$, by virtue of Corollary of Proposition 3.1. By Skorobod's theorem we can construct copies $\left(X_{k}^{*}, \mathscr{A}_{k}^{*}\right)$ and $\left(X^{*}, \mathscr{A}^{*}\right)$ of $\left(X_{k}, \mathscr{A}_{k}\right)$ and $(X, \mathscr{A})$ respectively, so that $\Omega_{k}^{*}=\Omega^{*}, F_{k}^{*}=F^{*}$, $\stackrel{P}{P}_{k}^{*}=\stackrel{P}{P}^{*}$ and $\stackrel{P}{P}^{*}$-almost surely $\left(X_{k}^{*}, \xi_{k}^{*}, B_{k}^{*}, Y_{k}^{*}, q_{k}^{*}\right) \rightarrow\left(X^{*}, \xi^{*}, B^{*}, Y^{*}, q^{*}\right)$ in $d_{T}$. For non-exceptional $\omega \in \Omega^{*}$, we put $\lambda_{k}=q_{k}^{*}(\cdot, \omega), y_{k}=Y_{k}^{*}(\cdot, \omega)$, $\lambda=q^{*}(, \omega)$ and $y=Y^{*}(, \omega)$. Then $y_{k}, \lambda_{k}, y$ and $\lambda$ satisfy the condition of Theorem 5, Theorefore we have

$$
\Lambda\left(\theta_{k}, Y_{k}^{*}, q_{k}^{*}, \nu_{k}\right) \longrightarrow \Lambda\left(\theta, Y^{*}, q^{*}, \nu\right), \quad \stackrel{P}{P}^{*} \text {-almost surely . }
$$

On the other hand Theorem 5 tells us that the mapping $\Lambda(\theta, \cdot, \cdot, \nu)$; $C\left(\left[\begin{array}{ll}0 & T\end{array}\right] \rightarrow R^{1}\right) \times\left(\hat{M}\left(\left[\begin{array}{ll}0 & T\end{array}\right] \times \Gamma\right)\right) \rightarrow M\left(R^{n}\right)$ is continuous. So $\Lambda(\theta, Y, q, \nu)$ is a random variable, i.e. $\Lambda(\theta, Y, q, \nu) \in m$. Consequently (5.31) implies (5.30).

Recalling Corollary of Theorem 4, we get

Corollary. For any $\mathscr{A} \in \mathfrak{Q}(\mu)$, there exists an approximate admissible switching system $\mathscr{A}_{k} \in \mathfrak{U}(\mu)$, such that

$$
\Lambda\left(\theta_{k}, Y_{k}, q_{k}, \nu\right) \longrightarrow \Lambda(\theta, Y, q, \nu) \quad \text { in metric } \delta
$$

where $\mu=\nu /\|\nu\|$.

\section{§6. Semigroup}

Let $C$ be the Banach lattice of the totality of bounded continuous mappings from $\left(M\left(R^{n}\right), \Delta\right)$ into $R^{1}$, with supremum norm and the order $\leq$, i.e.

$$
\phi \leq \psi \Longleftrightarrow \phi(\nu) \leq \psi(\nu) \quad \text { for } \forall_{\nu} \in M\left(R^{n}\right) .
$$

For $\nu \in M\left(R^{n}\right), \mathscr{A} \in \mathfrak{X}(\nu /\|\nu\|)$ and $\phi \in C$ we define $J$ by

$$
\begin{gathered}
J(t, \mathscr{A}, \nu, \phi)=\stackrel{\circ}{E}(\phi(\Lambda(t, Y, q, \nu)) \\
\stackrel{\circ}{E}[\stackrel{\circ}{E}(\phi(\Lambda(t, Y, q, \nu) / \sigma(Y, q)] \\
=\int_{C([0 T] \rightarrow R 1) \times \hat{M}([0 T] \times \Gamma)} \stackrel{\circ}{E}(\phi(\Lambda(t, y, \lambda, \nu)) \pi(d y, d \lambda)
\end{gathered}
$$


where $\pi$ is the probability distribution of $(Y, q)$. Since $\stackrel{\circ}{E}(\phi(\Lambda(t, y, \lambda, \nu))$ depends only on $\phi, \nu, t, y$ and $\lambda, J(t, \mathscr{A}, \nu, \phi)$ can be denoted by $J(t, \pi, \nu, \phi)$.

Define $S(t) \phi$ by

$$
S(t) \phi(\nu)=\sup _{\mathscr{A} \in \mathscr{\mathscr { U }}(\nu /\|\nu\|)} J(t, \mathscr{A}, \nu, \phi) .
$$

Then by Proposition 2.3 (ii) and (6.3) we have

$$
S(t) \phi(\nu)=\sup _{\pi \in \mathscr{F}} J(t, \pi, \nu, \phi) .
$$

Proposition 6.1. $J(t, \pi, \nu, \phi)$ is continuous in $(t, \pi, \nu) \in[0 T] \times \mathscr{P} \times$ $M\left(R^{n}\right)$.

Proof. Let $t_{k} \rightarrow t, \pi_{k} \rightarrow \pi$ and $\nu_{k} \rightarrow \nu$ in their topologies. Take $\mathscr{A}_{k} \in$ $\mathfrak{X}\left(\nu_{k} /\left\|\nu_{k}\right\|\right)$ (and $\left.\mathscr{A} \in \mathfrak{X}(\nu /\|\nu\|)\right)$ such that the probability distribution of $\left(y_{k}, q_{k}\right)$ (and $(Y, q)$ ) is $\pi_{k}$ (and $\pi$ respectively). Then $\mathscr{A}_{k} \rightarrow \mathscr{A}$ by Proposition 2.2. Therefore Theorem 6 guarantees that $\Lambda\left(t_{k}, \pi_{k}, \nu_{k}, \phi\right) \rightarrow \Lambda(t, \pi, \nu, \phi)$ in metric $\delta$ that is in the Prohorov topology. By Skorobod's theorem we can take a copy $\Lambda_{k}^{*}$ of $\Lambda\left(t_{k}, \pi_{k}, \nu_{k}, \phi\right)$ and $\Lambda^{*}$ of $\Lambda(t, \pi, \nu, \phi)$ so that $\Lambda_{k}^{*}$ converges to $\Lambda_{k}^{*}$ almost surely on $\left(\Omega^{*}, F^{*}, \dot{P}^{*}\right)$. Since $\phi$ is bounded continuous, we see that

$$
\left\{\begin{array}{l}
J\left(t_{k}, \pi_{k}, \nu_{k}, \phi\right)=\stackrel{\circ}{E}_{k} \phi\left(\Lambda\left(t_{k}, Y_{k}, q_{k}, \nu_{k}\right)=\stackrel{\circ}{E}^{*} \phi\left(\Lambda_{k}^{*}\right)\right. \\
J(t, \pi, \nu, \phi)=\stackrel{\circ}{E} \phi\left(\Lambda(t, Y, q, \nu)=\stackrel{\circ}{E}^{*} \phi\left(\Lambda^{*}\right)\right. \\
\stackrel{\circ}{E}^{*} \phi\left(\Lambda_{k}^{*}\right) \rightarrow \stackrel{\circ}{E}^{*} \phi\left(\Lambda^{*}\right) .
\end{array}\right.
$$

This completes the proof of Proposition 6.1.

Since $\mathscr{P}$ is a compact metric space by Proposition 2.3 (i), we can conclude the following proposition.

Proposition 6.2. $S(t) \phi \in C$ whenever $\phi \in C$. That is, $S(t)$ is a mapping from $C$ into $C$. Recalling Corollary of Theorem 6, we see

$$
S(t) \phi(\nu)=\sup _{\substack{\mathscr{L} \in \mathcal{R}(\nu /\|\nu\|) \\ \mathscr{A}: \text { swisching syst. }}} J(t, \mathscr{A}, \nu, \phi)
$$

THEOREM 7. $S(t+\theta)=S(t) S(\theta), S(0)=$ identity.

Proof. Consider the $\operatorname{SDE}$ on $(\Omega, F, \stackrel{\circ}{P})$, for $\lambda \in \hat{M}\left(\left[\begin{array}{ll}0 & T\end{array}\right] \times \Gamma\right)$

$$
\left\{\begin{array}{l}
d \eta(t)=\alpha(\eta(t)) d B(t)+\int_{\Gamma} \gamma(\eta(t), u) \lambda(d t, d u) \\
\eta(0)=\xi
\end{array}\right.
$$


Since a solution $\eta(\cdot, \xi, B, \lambda)$ is unique, $\eta$ satisfies the following relation

$$
\eta(t+\theta, \xi, B, \lambda)=\eta\left(\theta, \eta(t, \xi, B, \lambda), B_{t}^{+}, \lambda_{t}^{+}\right)
$$

where $B_{t}^{+}(s)=B(t+s)-B(t), \lambda_{t}^{+}(s, A)=\lambda(t+s, A)-\lambda(t, A)$.

Using Ito's formula we get

$$
\mathscr{L}(t+s, \xi, B, y, \lambda)=\mathscr{L}(t, \xi, B, y, \lambda) \mathscr{L}\left(s, \eta(t, \xi, B, \lambda), B_{t}^{+}, y_{t}^{+}, \lambda_{t}^{+}\right) .
$$

Define $v:\left[\begin{array}{ll}0 & T\end{array}\right] \times R^{n} \times C\left(\left[\begin{array}{ll}0 & T\end{array}\right] \rightarrow R^{1}\right) \times \hat{M}\left(\left[\begin{array}{ll}0 & T\end{array}\right] \times \Gamma\right) \times C_{b}\left(R^{n}\right) \rightarrow R^{1}$ by

$$
v(t, x, y, \lambda, f)=\stackrel{\circ}{E} f(\eta(t, x, B, \lambda)) \mathscr{L}(t, x, B, y, \lambda)
$$

where $\stackrel{E}{E}$ of the right side stands for the expectation with respect to $B$, since the starting point $x$ is not random. From (6.10) and (6.11) we have

$$
\begin{array}{rl}
v(t+s, x, y, \lambda, f)=\stackrel{\circ}{E} & f(\eta(t+s, x, B, \lambda)) \mathscr{L}(t+s, x, B, y, \lambda) \\
=\stackrel{\circ}{E}\left[\mathscr { L } ( t , x , B , y , \lambda ) \stackrel { \circ } { E } \left(f \left(\eta\left(s, \eta(t, x, B, \lambda), B_{t}^{+}, \lambda_{t}^{+}\right)\right.\right.\right. \\
\left.\times \mathscr{L}\left(s, \eta(t, x, B, \lambda), B_{t}^{+}, y_{t}^{+}, \lambda_{t}^{+} / \sigma_{t}(B)\right)\right] .
\end{array}
$$

Since $\eta(t, x, B, \lambda)$ is $\sigma_{t}(B)$-measurable, we see

$$
\begin{aligned}
& \stackrel{\circ}{E}\left(f\left(\eta\left(s, \eta(t, x, B, \lambda), B_{t}^{+}, \lambda_{t}^{+}\right) \mathscr{L}\left(s, \eta(t, x, B, \lambda), B_{t}^{+}, y_{t}^{+}, \lambda_{t}^{+}\right) / \sigma_{t}(B)\right)\right) \\
& \quad=v\left(s, \eta(t, x, B, \lambda), y_{t}^{+}, \lambda_{t}^{+}, f\right)
\end{aligned}
$$

and, combining with (6.12) we get

$$
\begin{aligned}
v(t+s, x, y, \lambda, f) & =\stackrel{\circ}{E} v\left(s, \eta(t, x, B, \lambda), y_{t}^{+}, \lambda_{t}^{+}, f\right) \mathscr{L}(t, x, B, y, \lambda) \\
& =v\left(t, x, y, \lambda, v\left(s, \cdot, y_{t}^{+}, \lambda_{t}^{+}, f\right)\right) .
\end{aligned}
$$

Recalling (5.8) and (5.15) we get

$$
\langle f, \Lambda(t, y, \lambda, \nu)\rangle=\langle v(t, \cdot, y, \lambda), \nu\rangle, \quad f \in C_{b}\left(R^{n}\right) .
$$

Hence, by (6.14), we have

$$
\begin{aligned}
\langle f, \Lambda(t+s, y, \lambda, \nu)\rangle & =\langle v(t+s, \cdot, y, \lambda), \nu\rangle \\
& =\left\langle v\left(t, \cdot, y, \lambda, v\left(s, \cdot, y_{t}^{+}, \lambda_{t}^{+}, f\right)\right), \nu\right\rangle \\
& =\left\langle v\left(s, \cdot, y_{t}^{+}, \lambda_{t}^{+}, f\right), \Lambda(t, y, \lambda, \nu)\right\rangle \\
& =\left\langle f, \Lambda\left(s, y_{t}^{+}, \lambda_{t}^{+}, \Lambda(t, y, \lambda, \nu)\right)\right\rangle, \quad f \in C_{b}\left(R^{n}\right) .
\end{aligned}
$$

Consequently

$$
\Lambda(t+s, y, \lambda, \nu)=\Lambda\left(s, y_{t}^{+}, \lambda_{t}^{+}, \Lambda(t, y, \lambda, \nu)\right) .
$$

Since (6.17) holds for any $y \in C\left(\left[\begin{array}{ll}0 & T\end{array}\right] \rightarrow R^{1}\right)$ and $\left.\lambda \in \hat{M}(0 T] \times \Gamma\right)$, we have, for any $\mathscr{A}=(\Omega, F, \stackrel{\circ}{P}, \xi, B, Y, q) \in \mathfrak{A}(\nu /\|\nu\|)$, 


$$
\Lambda(t+s, Y, q, \nu)=\Lambda\left(s, Y_{t}^{+}, q_{t}^{+}, \Lambda(t, Y, q, \nu)\right), \stackrel{P}{P} \text {-almost surely } .
$$

This implies

$$
\begin{aligned}
J(t+s, \mathscr{A}, \nu, \phi) & =\stackrel{\circ}{E} \phi(\Lambda(t+s, Y, q, \nu)) \\
& =\stackrel{\circ}{E}\left[\stackrel{\circ}{E}\left(\phi\left(\Lambda(t+s, Y, q, \nu) / \sigma_{T}(Y q)\right)\right)\right] \\
& =\stackrel{\circ}{E} \phi\left(\Lambda\left(s, Y_{t}^{+}, q_{t}^{+}, \Lambda(t, Y, q, \nu)\right)\right) \\
& =\stackrel{\circ}{E} \stackrel{\circ}{E}\left(\phi\left(\Lambda\left(s, Y_{t}^{+}, q_{t}^{+}, \Lambda(t, Y, q, \nu)\right) / \sigma_{t}(Y, q)\right)\right) .
\end{aligned}
$$

Under the regular conditional probability $\stackrel{\circ}{P}\left(/ \sigma_{t}(Y, q)\right),\left(B_{t}^{+}, Y_{t}^{+}\right)$is $a(n+1)$ dimensional Brownian motion, $\left(Y_{t}^{+}, q_{t}^{+}\right)$independent of $(B, \xi)$ and $Y_{t}^{+}$is $\sigma_{s}\left(Y_{t}^{+}, q_{t}^{+}\right)$-Brownian motion, i.e. independent increments. Hence $\eta(t, \xi$, $B, q), B_{t}^{+}$and $\left(Y_{t}^{+}, q_{t}^{+}\right)$are independent under conditional probability $\stackrel{P}{P}\left(\cdot / \sigma_{t}(Y, q)\right), \stackrel{\circ}{P}$-almost surely, although the probability distribution of $q_{t}^{+}$ might depend on the past value of $(Y(\theta), q(\theta, A), \theta \leq t, A \in B(\Gamma))$. Hence there exists a null set $N \in \sigma_{t}(Y, q)$, such that for $\omega \notin N,\left(\Omega, F, \stackrel{\circ}{P}\left(\cdot / \sigma_{t}(Y, q)\right)\right.$ $\left.(\omega), \eta(t, \xi, B, q), B_{t}^{+}, Y_{t}^{+}, q_{t}^{+}\right) \in \mathfrak{A}$. Therefore

$$
\begin{array}{r}
\stackrel{\circ}{E}\left(\phi\left(\Lambda\left(s, Y_{t}^{+}, q_{t}^{+}, \Lambda(t, Y, q, \nu)\right) / \sigma_{t}(Y, q)\right)\right) \leq(S(s) \phi)(\Lambda(t, Y, q, \nu)), \\
\dot{P} \text {-almost surely } .
\end{array}
$$

Combining (6.20) with (6.19), we have

$$
J(t+s, \mathscr{A}, \nu, \phi) \leq S(t)(S(s) \phi)(\nu) .
$$

Taking the supremum with respect to $\mathscr{A} \in \mathfrak{A}(\nu /\|\nu\|)$, we have

$$
S(t+s) \phi(\nu) \leq S(t)(S(s) \phi)(\nu)
$$

For the converse inequality we will show some lemmas

Lemma 1. Let $N \subset\left(M\left(R^{n}\right), \Delta\right)$ be totally bounded. Then $\left\{\Lambda\left(t, Y_{\mathscr{A}}, q_{\mathscr{A}}, \nu\right)\right.$; $\mathscr{A} \in \mathfrak{X}(\nu /\|\nu\|), \nu \in N\}$ is totally bounded in $(m, \delta)$.

Proof. Consider the SDE, for $\mathscr{A}=(\Omega, F, \stackrel{\circ}{P}, \xi, B, Y, q) \in \mathfrak{X}(\nu /\|\nu\|)$.

$$
\left\{\begin{array}{l}
d \eta(t)=\alpha(\eta(t)) d B(t)+\int_{\Gamma} \gamma(\eta(t), u) \lambda(d t, d u) \\
\eta(0)=\xi .
\end{array}\right.
$$

Then, using this unique solution $\eta(t)=\eta(t, \xi, B, \lambda)$ we have

$$
\Lambda(t, y, \lambda, \nu)(A)=\|\nu\| \stackrel{\circ}{E} \chi_{A}(\eta(t)) \mathscr{L}(t, \xi, B, y, \lambda) .
$$

Hence, by (5.7) 


$$
\begin{aligned}
A^{2}(t, y, \lambda, \nu)(A) & \leq\|\nu\|^{2} \stackrel{\circ}{P}(\eta(t) \in A) \stackrel{\circ}{E} \mathscr{L}^{2}(t, \xi, B, y, \lambda) \\
& \leq\|\nu\|^{2} \stackrel{\circ}{P}(\eta(t) \in A) \exp \left[2 K_{2}(t+1)\left(\|y\|_{t}+1\right)^{2}\right]
\end{aligned}
$$

where $\|y\|_{t}=\sup _{s \leq t}|y(s)|$. This means

$$
\begin{array}{r}
\Lambda(t, y, \lambda, \nu)(A) \leq\|\nu\| \sqrt{\dot{P}(\eta(t) \in A)} \exp \left[K_{2}(t+1)(1+a)^{2}\right], \\
\text { whenever }\|y\|_{t} \leq a .
\end{array}
$$

On the other hand the condition (A1) implies that, for $\varepsilon^{\prime}>0$, there exists $b=b\left(\varepsilon^{\prime}, t, N\right)$ such that

$$
\stackrel{\circ}{P}(|\eta(t, \xi, B, \lambda)|>b)<\varepsilon^{\prime} \quad \text { for } \forall \lambda \in \hat{M}([0 T] \times \Gamma), \mathscr{A} \in \mathfrak{A}\left(\frac{\nu}{\|\nu\|}\right), \nu \in N .
$$

Since $Y$ is a Brownian motion, for $\varepsilon>0$ there exists $a=a(\varepsilon)$ such that

$$
\stackrel{\circ}{P}\left(\sup _{s \leq t}|Y(s)| \leq a\right)>1-\varepsilon \quad \text { for } \forall \mathscr{A} \in \mathfrak{A} .
$$

Putting $\varepsilon^{\prime}=\varepsilon^{2} e^{-2 K_{2}(1+t)(1+a(\varepsilon))^{2}},(6.26)$ gives

$$
\Lambda(t, y, \lambda, \nu)\left(K^{c}\right)<\varepsilon\|\nu\| \quad \text { for } \lambda \in \hat{M}\left(\left[\begin{array}{ll}
0 & T
\end{array}\right] \times \Gamma\right)
$$

whenever $\|y\|_{t}<a$, where the compact set $K$ is given by

$$
K=\left\{x \in R^{n}:|x| \leq b\left(\varepsilon^{\prime}, t, N\right)\right\} .
$$

Therefore combining (6.28) and (6.29), we see, for $\mathscr{A} \in \mathfrak{R}(\nu /\|\nu\|)$,

$$
1-\varepsilon<\stackrel{\circ}{P}\left(\sup _{s \leq t}|Y(s)| \leq a(\varepsilon)\right) \leq \stackrel{P}{P}\left(\Lambda(t, Y, q, \nu)\left(K^{c}\right)<\varepsilon\|\nu\|\right) .
$$

Recalling the condition " $0<c^{\prime} \leq\|\nu\| \leq c$ for $\forall_{\nu} \in N$ ", (6.31) implies Lemma 1 by virtue of Proposition 5.1.

Applying Prohorov's theorem, Lemma 1 gives

Lemma 2. For $\varepsilon>0$ and a totally bounded set $N \subset\left(M\left(R^{n}\right), \Delta\right)$ there exists a compact set $\tilde{N}=\tilde{N}(\varepsilon, t, N) \subset\left(M\left(R^{n}\right), \Delta\right)$ such that

$$
\stackrel{\circ}{P}(\Lambda(t, Y, q, \nu) \in \tilde{N})>1-\varepsilon \quad \text { for } \mathscr{A} \in \mathfrak{A}\left(\frac{\nu}{\|\nu\|}\right), \nu \in N
$$

LEMma 3. Suppose that $M\left(R^{n}\right)=M_{0} \cup \cdots \cup M_{\ell}$ is a Borel partition of $M\left(R^{n}\right)$. Let $\nu_{i} \in M_{i}$ and $\mathscr{A}=\left(\Omega_{i}, F_{i}, \stackrel{P}{P}_{i}, \xi_{i}, B_{i}, Y_{i}, q_{i}\right) \in \mathfrak{A}\left(\nu_{i} /\left\|\nu_{i}\right\|\right)$. For any fixed $\mathscr{A}=(\Omega, F, P, \xi, B, Y, q) \in \mathfrak{U}(\nu /\|\nu\|)$ we define $\tilde{\Omega}, \tilde{F}, \tilde{P}, \tilde{\xi}, \tilde{B}, \tilde{Y}, \tilde{q}$ as follows. 


$$
\begin{aligned}
& \tilde{\Omega}=\Omega \times \Omega_{0} \times \Omega_{1} \times \cdots \times \Omega_{\ell}, \quad \tilde{F}=F \times F_{0} \times \cdots \times F_{\ell} \\
& \tilde{\tilde{P}}=\dot{P} \times \dot{P}_{1} \times \cdots \times \dot{P}_{\ell}, \quad \tilde{\xi}=\xi \\
& \tilde{B}(\theta)=\left\{\begin{array}{l}
B(\theta), \quad \theta \leq t \\
B(t)+\sum_{i=0}^{\ell} B_{i}(\theta-t) \chi_{M_{i}}(\Lambda(t, Y, q, \nu)), \quad \theta \geq t .
\end{array}\right.
\end{aligned}
$$

$\tilde{Y}$ is defined in the same way.

$$
\tilde{q}(\theta, A)=\left\{\begin{array}{l}
q(\theta, A), \quad \theta \leq t \\
q(t, A)+\sum_{i=0}^{\ell} q_{i}\left(\theta_{i}-t, A\right) \chi_{M_{i}}(\Lambda(t, Y, q, \nu)), \quad \theta \geq t .
\end{array}\right.
$$

Then $\tilde{\mathscr{A}}=(\tilde{\Omega}, \tilde{F}, \tilde{\check{P}}, \tilde{\xi}, \tilde{B}, \tilde{Y}, \tilde{q}) \in \mathfrak{U}(\nu /\|\nu\|)$.

Proof. $\xi$ is independent of $\left\{(B, Y, q),\left(\xi_{i}, B_{i}, Y_{i}, q_{i}\right), i=0, \cdots, \ell\right\}$. So $\tilde{\xi}$ is independent of $(\tilde{B}, \tilde{Y}, \tilde{q})$.

$\tilde{B}$ is a Brownian motion, because for $g \in C_{b}\left(\left(R^{n}\right)^{k}\right)$ and $\theta_{j} \geq t, j=$ $1, \cdots, k$,

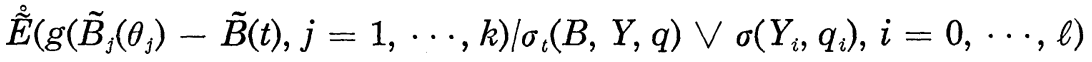

$$
\begin{aligned}
& =\stackrel{\circ}{E}_{i}\left(g\left(B_{i}\left(\theta_{j}-t\right), j=1, \cdots, k\right) \quad \text { if } \Lambda(t, Y, q, \nu) \in M_{i} .\right.
\end{aligned}
$$

Hence $(\tilde{B}(s)-\tilde{B}(t), s \geq t)$ is a Brownian motion which is independent of $\sigma_{t}(B, Y, q) \vee \sigma\left(Y_{0}, q_{0}\right) \vee \cdots \vee \sigma\left(Y_{\ell}, q_{\ell}\right)$, since $B_{i}$ is a Brownian motion. This implies that $(\tilde{B}(s)-\tilde{B}(t), s \geq t, B(\theta), \theta \leq t)$ is independent of $((Y, q)$, $\left.\left(Y_{i}, q_{i}\right), i=0, \cdots, \ell\right)$, since $B$ is independent of $\left((Y, q),\left(Y_{i}, q_{i}\right), i=0, \cdots, \ell\right)$. Therefore $\tilde{B}$ is independent of $(\tilde{Y}, \tilde{q})$, because $(\tilde{Y}, \tilde{q})$ is measurable with respect to $\sigma\left(Y, q, Y_{0}, q_{0}, \cdots, Y_{\ell}, q_{\ell}\right)$.

Using a similar calculation as (6.33), we see that for $g \in C_{b}\left(R^{k}\right)$ and $\theta_{j} \geq \theta \geq t, j=1, \cdots, k$

$$
\begin{aligned}
& \stackrel{\check{E}}{(}\left(g\left(\tilde{Y}\left(\theta_{j}\right)-\tilde{Y}(\theta), j=1, \cdots, k / \sigma_{t}(Y, q) \vee \sigma_{\theta-t}\left(Y_{0}, q_{0}, \cdots, Y_{\ell}, q_{\ell}\right)\right)\right) \\
& \quad=\dot{E}_{i}\left(g\left(Y_{i}\left(\theta_{j}\right)-Y_{i}(\theta), j=1, \cdots, k\right)\right), \quad \text { if } \Lambda(t, Y, q, \nu) \in M_{i} .
\end{aligned}
$$

Therefore $(\tilde{Y}(s)-\tilde{Y}(\theta), s \geq \theta)$ is a Brownian motion which is independent of $\sigma_{\theta}(\tilde{Y}, \tilde{q})$.

It is clear that $\tilde{q}$ satisfies the conditions (v) and (vi), from the definition of $\tilde{q}$. This completes the proof of Lemma 3 .

Now we prove the inequality (6.35) for Theorem.

$$
S(t+s) \phi(\nu) \geq S(t)(S(s) \phi)(\nu) .
$$


Since $J(s, \pi, \nu, \phi)$ is continuous in $\pi, \nu$ and $\mathscr{P}$ is compact, $J(s, \pi, \nu, \phi)$ is continuous in $\nu$ uniformly in $\pi \in \mathscr{P}$, namely for $\varepsilon>0$ there exists $\delta(\varepsilon, \nu)=$ $\delta(\varepsilon, \nu, s, \phi)$ such that, if $\Delta\left(\nu^{\prime}, \nu\right)>\delta(\varepsilon, \nu)$ then

$$
\left|J\left(s, \pi, \nu^{\prime}, \phi\right)-J(s, \pi, \nu, \phi)\right|<\varepsilon .
$$

Hence

$$
\left|S(s) \phi\left(\nu^{\prime}\right)-S(s) \phi(\nu)\right|<\varepsilon, \quad \text { if } \Delta\left(\nu^{\prime}, \nu\right)<\delta(\varepsilon, \nu) .
$$

Applying Lemma 2 for $N=\{\nu\}$, given $\varepsilon>0$ we can take a compact set $\tilde{N}$ as in (6.32). From (6.36) and (6.37) we can take a Borel partition of $\tilde{N}$, say $\tilde{N}=M_{1} \cup \cdots \cup M_{\ell}$, such that, if $\nu^{\prime}, \nu^{\prime \prime} \in M_{i}$, then

$$
\left|J\left(s, \pi, \nu^{\prime}, \phi\right)-J\left(s, \pi, \nu^{\prime \prime}, \phi\right)\right|<\varepsilon
$$

and

$$
\left|S(s) \phi\left(\nu^{\prime}\right)-S(s) \phi\left(\nu^{\prime \prime}\right)\right|<\varepsilon .
$$

Fix $\nu_{i} \in M_{i}, i=1, \cdots, \ell$, arbitrarily and take $\mathscr{A}_{i} \in \mathfrak{Y}\left(\nu_{i} /\left\|\nu_{i}\right\|\right)$ such that

$$
J\left(s, \pi_{i}, \nu_{i}, \phi\right) \geq S(s) \phi\left(\nu_{i}\right)-\varepsilon
$$

where $\pi_{i}=$ probability distribution of $\left(Y_{\star_{i}}, q_{\mathscr{A}_{i}}\right)$. Then, by $(6.38) \sim(6.40)$, we see

$$
\begin{array}{r}
J\left(s, \pi_{i}, \nu^{\prime}, \phi\right) \geq J\left(s, \pi_{i}, \nu_{i}, \phi\right)-\varepsilon \geq S(s) \phi\left(\nu_{i}\right)-2 \varepsilon \geq S(s) \phi\left(\nu^{\prime}\right)-3 \varepsilon \\
\text { for } \nu^{\prime} \in M_{i} .
\end{array}
$$

Let $\mathscr{A} \in \mathfrak{X}(\nu /\|\nu\|)$ and $M_{0}=\tilde{N}^{c}$ and take $\nu_{0} \in M_{0}\left(R^{n}\right)$ and $\mathscr{A}_{0} \in \mathfrak{Y}\left(\nu_{0} /\left\|\nu_{0}\right\|\right)$ arbitrarily. Then $M_{i}, i=0, \cdots, l$ is a Borel partition of $M\left(R^{n}\right)$. According to Lemma 3 we have $(\tilde{\Omega}, \tilde{F}, \stackrel{\mathscr{P}}{\tilde{\xi}}, \tilde{\xi}, \tilde{B}, \tilde{Y}, \tilde{q}) \in \mathfrak{X}(\nu /\|\nu\|)$. Then

$$
\begin{aligned}
& J(t+s, \tilde{\mathscr{A}}, \nu, \phi)=\stackrel{\stackrel{\circ}{E}}{\phi}(\Lambda(t+s, \tilde{Y}, \tilde{q}, \nu)) \\
& =\stackrel{\mathscr{\Xi}}{E}_{\phi}\left(\Lambda\left(s, \tilde{Y}_{t}^{+}, \tilde{q}_{t}^{+}, \Lambda(t, Y, q, \nu)\right)\right. \\
& =\stackrel{\check{E}}{\tilde{E}}\left[\stackrel{\circ}{E}\left(\phi\left(\Lambda\left(s, \tilde{Y}_{t}^{+}, \tilde{q}_{t}^{+}, \Lambda(t, Y, q, \nu)\right) / \sigma_{t}(Y, q)\right)\right]\right. \text {. }
\end{aligned}
$$

On the other hand, by (6.41) we have

$$
\begin{aligned}
& \stackrel{\check{E}}{\tilde{E}}\left(\phi\left(\Lambda\left(s, \tilde{Y}_{t}^{+}, \tilde{q}_{t}^{+}, \Lambda(t, Y, q, \nu)\right) / \sigma_{t}(Y, q)\right)\right) \\
& =\sum_{i=0}^{\ell}\left[\stackrel{\circ}{E}_{i} \phi\left(\Lambda\left(s, Y_{i}, q_{i}, \Lambda(t, Y, q, \nu)\right)\right] \chi_{M_{i}}(\Lambda(t, Y, q, \nu))\right. \\
& =\sum_{i=0}^{\ell} J\left(s, \mathscr{A}_{i}, \Lambda(t, Y, q, \nu)\right) \chi_{M_{i}}(\Lambda(t, Y, q, \nu))
\end{aligned}
$$




$$
\begin{aligned}
& \geq \sum_{i=0}^{\ell} S(s) \phi(\Lambda(t, Y, q, \nu)) \chi_{M_{i}}(\Lambda(t, Y, q, \nu))-3 \varepsilon-\|\phi\| \chi_{M_{0}}(\Lambda(t, Y, q, \nu)) \\
& =S(s) \phi(\Lambda(t, Y, q, \nu))-3 \varepsilon-\|\phi\| \chi_{M_{0}}(\Lambda(t, Y, q, \nu)) .
\end{aligned}
$$

Combining with (6.42) we see

$$
\begin{aligned}
J(t+s, \tilde{\mathscr{A}}, \nu, \phi) & \geq \stackrel{\circ}{E}(S(s) \phi)(\Lambda(t, Y, q, \nu))-3 \varepsilon-\|\phi\| \stackrel{P}{P}(\Lambda(t, Y, q, \nu) \notin \tilde{N}) \\
& \geq \stackrel{\circ}{E}(S(s) \phi)(\Lambda(t, Y, q, \nu))-3 \varepsilon-\|\phi\| \varepsilon .
\end{aligned}
$$

Since $\tilde{\mathscr{A}} \in \mathfrak{U}(\nu /\|\nu\|)$ we see

$$
S(t+s) \phi(\nu) \geq \stackrel{\circ}{E}(S(s) \phi)(\Lambda(t, Y, q, \nu))-\varepsilon(3+\|\phi\|) .
$$

Taking the supremum with respect to $\mathscr{A} \in \mathfrak{A}(\nu /\|\nu\|)$, we conclude

$$
S(t+s) \phi(\nu) \geq S(t)(S(s) \phi)(\nu)-\varepsilon(3+\|\phi\|) .
$$

Tending $\varepsilon \downarrow 0$, we get our desired inequality (6.35). This completes the proof of Theorem 7 .

\section{§7. Generator and properties of $S(t)$.}

We can easily see:

Proposition 7.1. The following properties hold,

(i) monotone, $S(t) \phi \leq S(t) \psi$ whenever $\phi \leq \psi$

(ii) contraction, $\|S(t) \phi-S(t) \psi\| \leq\|\phi-\psi\|$

(iii) continuity, $S(\theta) \phi(\nu) \rightarrow S(t) \phi(\nu)$ as $\theta \rightarrow t$,

uniformly on any compact set of $M\left(R^{n}\right)$.

That is, $S(t)$ is a monotone contraction weakly continuous semigroup on $C$.

Proof. (i ) From the definition of $J$, (7.1) is clear

$$
J(t, \pi, \nu, \phi) \leq J(t, \pi, \nu, \psi), \quad \text { if } \phi \leq \psi .
$$

Hence taking the supremum with respect to $\pi \in \mathscr{P}$, we have (i).

(ii) $|S(t) \phi(\nu)-S(t) \psi(\nu)|$

$$
\begin{aligned}
& \leq \sup _{\mathscr{A} \in \mathfrak{U}(\nu /\|\nu\|)}\left|\stackrel{\circ}{E} \phi\left(\Lambda\left(t, Y_{\mathscr{A}}, q_{\mathscr{A}}, \nu\right)\right)-\stackrel{\circ}{E} \psi\left(\Lambda\left(t, Y_{\mathscr{A}}, q_{\mathscr{A}}, \nu\right)\right)\right| \\
& \leq\|\phi-\psi\| .
\end{aligned}
$$

Hence taking the supremum with respect to $\nu \in M\left(R^{n}\right)$, we have (ii).

(iii) By Proposition 6.1. $J(t, \pi, \nu, \phi)$ is continuous in $(t, \pi, \nu) \in\left[\begin{array}{ll}0 & T\end{array}\right]$ $\times \mathscr{P} \times M\left(R^{n}\right)$. Since $\mathscr{P}$ is compact, $S(t) \phi(\nu)$ is continuous in $(t, \nu)$. Hence it is uniformly continuous on $[0 T] \times F$ where $F$ is compact in $M\left(R^{n}\right)$. This implies (iii). 
Now we calculate the generator of $S(t)$, according to [6]. We introduce the following set $\mathscr{D}$ of functions $\phi$ which depend on finitely many scalar products. Fix $H_{N} \in C^{\infty}([0 \infty) \rightarrow[0,1])$ such that $H_{N}(x)=1$ on $[0 N]$, $=0$ on $[N+1, \infty)$ and decreasing in $x$.

$$
\begin{aligned}
\mathscr{D}= & \left\{\phi ; M\left(R^{n}\right) \longrightarrow R^{1} ; \phi(\nu)=F\left(\left\langle f_{1}, \nu\right\rangle, \cdots,\left\langle f_{\ell}, \nu\right\rangle\right) H_{N}(\langle 1, \nu\rangle)\right. \\
& \text { with } \left.F \in C_{b}^{\infty}\left(R^{e}\right), f_{1}, \cdots, f_{\ell} \in C_{0}^{\infty}\left(R^{n}\right), \ell=1,2, \cdots, N=1,2, \cdots\right\}
\end{aligned}
$$

where $C_{0}^{\infty}$ denotes the space of $C^{\infty}$ functions with compact supports and $C_{b}^{\infty}$ the space of $C^{\infty}$ functions with any bounded derivative. Clearly $\mathscr{D} \subset C$. Moreover we have

Proposition 7.2. For $\phi \in C$ there exists $\Phi_{k} \in \mathscr{D}$ such that $\Phi_{k}(\nu) \underset{k \rightarrow \infty}{\rightarrow} \phi(\nu)$ for any $\nu \in M\left(R^{n}\right)$.

Proof. We can apply the same method as [6]. Let $v\left(x_{i}, 2^{-N}\right)$ (=open ball with center $x_{i}$, radius $\left.2^{-N}\right) i=1,2, \cdots$ be a covering of $R^{n}$ with $\bigcup_{i=1}^{k_{N}} v\left(x_{i}, 2^{-N}\right) \supset[-N, N]^{n}$ (say $\left.I_{N}\right)$. Let $g_{\ell}^{N}, \ell=1,2, \cdots$ be a $C^{\infty}$-partition of unity such that

$$
\begin{gathered}
\text { supp } g_{\ell}^{N} \subset v\left(x_{i}, 2^{-N}\right) \text { for some } i \\
\sum_{\ell=1}^{P_{N}} g_{\ell}=1 \quad \text { on } I_{N} .
\end{gathered}
$$

Take $y_{\ell}^{N} \in \operatorname{supp} g_{\ell}^{N} \cap I_{N}$ arbitrarily. Putting $c_{\ell}^{N}(\nu)=\left\langle g_{\ell}^{N}, \nu\right\rangle$, we define $\nu_{N}$ by

$$
\nu_{N}=\sum_{\ell=1}^{P_{N}} c_{\ell}^{N}(\nu) \delta_{y_{\ell}^{N}} .
$$

Then $\|\nu\| \geq\left\|\nu_{N}\right\| \geq \nu\left(I_{N}\right)$ and for $g \in C_{b}\left(R^{n}\right)$

$$
\left\langle g, \nu_{N}\right\rangle \rightarrow\langle g, \nu\rangle, \quad \text { as } N \rightarrow \infty \text {. }
$$

Hence

$$
\Delta\left(\nu_{N}, \nu\right) \rightarrow 0, \text { as } N \rightarrow \infty .
$$

Denote $F_{N}\left(z_{1}, \cdots, z_{p_{N}}: \phi\right)=\phi\left(\sum_{\ell=1}^{p_{N}} z_{\ell} \delta_{y_{\ell}}\right)$. That is,

Therefore, by (7.6), we have

$$
\phi\left(\nu_{N}\right) \rightarrow \phi(\nu) \text { as } N \rightarrow \infty .
$$

From the definition of $H_{N}, \lim _{N \rightarrow \infty} H_{N}(\langle 1, \nu\rangle)=1$ for any $\nu \in M\left(R^{n}\right)$. Hence 
(7.9) gives

$$
\left.F_{N}\left(\left\langle g_{1}^{N}, \nu\right\rangle, \cdots, g_{p_{N}}^{N}, \nu\right\rangle: \phi\right) H_{N}(\langle 1, \nu\rangle) \rightarrow \phi(\nu), \quad \text { as } \quad N \rightarrow \infty .
$$

Take $\tilde{F}_{N} \in C_{b}^{\infty}\left(R^{p_{N}}\right)$ such that

$$
\left\|\tilde{F}_{N}-F_{N}\right\|_{L_{\infty}\left(I_{N+1}\right)}<2^{-N}
$$

Then we have

$$
\begin{aligned}
& \mid \tilde{F}_{N}\left(\left\langle g_{1}^{N}, \nu\right\rangle, \cdots,\left\langle g_{p_{N}}^{N}, \nu\right\rangle\right) H_{N}(\langle 1, \nu\rangle) \\
& \quad-F_{N}\left(\left\langle g_{1}^{N}, \nu\right\rangle, \cdots,\left\langle g_{p_{N}}^{N}, \nu\right\rangle: \phi\right) H_{N}(\langle 1, \nu\rangle) \mid<2^{-N} .
\end{aligned}
$$

Combining with (7.10), we complete the proof.

We calculate the generator of $S(t)$, recalling (6.7). For an admissible switching system $\mathscr{A} \in \mathfrak{A}(\nu /\|\nu\|)$, we have the following Zakai equation for $\Lambda(t)=\Lambda\left(t, Y_{\mathscr{A}}, q_{\mathscr{A}}, \nu\right)$, (see Theorem 5.2 in [8]),

$$
\left\{\begin{array}{l}
d\langle f, \Lambda(t)\rangle=\langle A(U(t)) f, \Lambda(t)\rangle d t+\langle h f, \Lambda(t)\rangle d Y(t) \\
\langle f, \Lambda(0)\rangle=\langle f, \nu\rangle \quad \text { for } f \in C_{b}^{2}\left(R^{n}\right),
\end{array}\right.
$$

where

$$
Y=Y_{\&}, \quad A(u)=\sum_{i j} a_{i j}(x) \frac{\partial^{2}}{\partial x_{i} \partial x_{j}}+\sum_{i} \gamma_{i}(x . y) \frac{\partial}{\partial x_{i}}
$$

and $U(t)$ is a $\sigma_{t}(q)$-progressively measurable process for $q$ (see Theorem 4 ).

Therefore using a routine method we have

$$
\begin{gathered}
\stackrel{\circ}{E}|\langle f, \Lambda(t)\rangle-\langle f, \nu\rangle|^{2} \leq K_{3}(t+1) \int_{0}^{t} \stackrel{\circ}{E}\langle 1, \Lambda(\theta)\rangle^{2} d \theta \\
\stackrel{\circ}{E}\langle 1, \Lambda(t)\rangle=\langle 1, \nu\rangle=\|\nu\|
\end{gathered}
$$

and

$$
\stackrel{\circ}{E}\|\Lambda(t)\|^{2} \leq\|\nu\|^{2} e^{K_{4} t}
$$

where $K_{3}$ and $K_{4}$ are independent of $\mathscr{A}$. Combining these evaluations, we have

$$
\stackrel{\circ}{E}|\langle f, \Lambda(t)\rangle,-\langle f, \nu\rangle|^{2} \leq K_{2}\|\nu\|^{2}(t+1) t e^{K_{4} t} .
$$

Let $\Phi \in \mathscr{D}$, say $\Phi(\nu)=F\left(\left\langle f_{1}, \nu\right\rangle, \cdots,\left\langle f_{\ell}, \nu\right\rangle\right) H_{N}(\langle 1, \nu\rangle)$. For simplicity we put $f_{0}=1$, and $\tilde{F}\left(z_{0}, z_{1}, \cdots, z_{\ell}\right)=F\left(z_{1}, \cdots, z_{\ell}\right) H_{N}\left(z_{0}\right)$. Appealing to Ito's formula, we see 


$$
\begin{aligned}
& d \Phi(\Lambda(t))=\sum_{i=0}^{\ell} \frac{\partial \tilde{F}}{\partial z_{i}}\left(\left\langle f_{0}, \Lambda(t)\right\rangle, \cdots,\left\langle f_{\ell}, \Lambda(t)\right\rangle\right)\left\langle A(U(t)) f_{i}, \Lambda(t)\right\rangle d t \\
& +\frac{1}{2} \sum_{i j=0}^{\ell} \frac{\partial^{2} \tilde{F}}{\partial z_{i} \partial z_{j}}\left(\left\langle f_{0}, \Lambda(t)\right\rangle, \cdots,\left\langle f_{\ell}, \Lambda(t)\right\rangle\right)\left\langle h f_{i}, \Lambda(t)\right\rangle\left\langle h f_{j}, \Lambda(t)\right\rangle d t \\
& \quad+\sum_{i=0}^{\ell} \frac{\partial \tilde{F}}{\partial z_{i}}\left(\left\langle f_{0}, \Lambda(t)\right\rangle, \cdots,\left\langle f_{\ell}, \Lambda(t)\right\rangle\right)\left\langle h f_{i}, \Lambda(t)\right\rangle d Y(t) .
\end{aligned}
$$

Using (7.16) we have, for $s \leqq 1$,

$$
\begin{aligned}
\int_{0}^{s} \stackrel{\circ}{E} \mid \frac{\partial \tilde{F}}{\partial z_{i}}\left(\left\langle f_{0}, \Lambda(t)\right\rangle, \cdots,\left\langle f_{\ell}, \Lambda(t)\right\rangle\right)\left\langle A(U(t)) f_{i}, \Lambda(t)\right\rangle \\
\quad-\frac{\partial \tilde{F}}{\partial z_{i}}\left(\left\langle f_{0}, \nu\right\rangle, \cdots,\left\langle f_{\ell}, \nu\right\rangle\right)\left\langle A(U(t)) f_{i}, \nu\right\rangle \mid d t \leq K_{5}\|\nu\| s^{3 / 2}
\end{aligned}
$$

and

$$
\begin{aligned}
\int_{0}^{s} \stackrel{\circ}{E} \mid & \frac{\partial^{2} \tilde{F}}{\partial z_{i} \partial z_{j}}\left(\left\langle f_{0}, \Lambda(t)\right\rangle, \cdots,\left\langle f_{\ell}, \Lambda(t)\right\rangle\right)\left\langle h f_{i}, \Lambda(t)\right\rangle\left\langle h f_{j},(t)\right\rangle \\
& -\frac{\partial^{2} \tilde{F}}{\partial z_{i} \partial z_{j}}\left(\left\langle f_{0}, \nu\right\rangle, \cdots,\left\langle f_{\ell}, \nu\right\rangle\right)\left\langle h f_{i}, \nu\right\rangle\left\langle h f_{j}, \nu\right\rangle \mid d t \leq K_{5}\|\nu\| s^{3 / 2}
\end{aligned}
$$

with $K_{5}$ which is independent of $\mathscr{A}$ and $\nu$.

Define $G \Phi$ as follows

$$
\begin{aligned}
G \Phi(\nu)= & \sup _{u \in \Gamma}\left(\sum_{i=0}^{\ell} \frac{\partial \tilde{F}}{\partial z_{i}}\left(\left\langle f_{0}, \nu\right\rangle, \cdots,\left\langle f_{\ell}, \nu\right\rangle\right)\left\langle A(u) f_{i}, \nu\right\rangle\right) \\
& +\frac{1}{2} \sum_{i j=0}^{\ell} \frac{\partial^{2} \tilde{F}}{\partial z_{i} \partial z_{j}}\left(\left\langle f_{0}, \nu\right\rangle, \cdots,\left\langle f_{\ell}, \nu\right\rangle\right)\left\langle h f_{i}, \nu\right\rangle\left\langle h f_{j}, \nu\right\rangle .
\end{aligned}
$$

Since $\tilde{F}$ is smooth and $\left\langle A(u) f_{i}, \nu\right\rangle$ continuous in $u$ and $\nu, G \Phi(\nu)$ is continuous in $\nu$. Moreover $G \Phi(\nu)=0$ whenever $\|\nu\| \geq N+1$. Therefore $G \Phi$ is bounded. This implies that $G \Phi \in C$ for $\Phi \in \mathscr{D}$.

We remark that

$$
\begin{aligned}
& \sum_{i} \int_{0}^{s} \stackrel{\circ}{E} \frac{\partial \tilde{F}}{\partial z_{i}}\left(\left\langle f_{0}, \nu\right\rangle, \cdots,\left\langle f_{\ell}, \nu\right\rangle\right)\left\langle A\left(U(t) f_{i}, \nu\right\rangle d t\right. \\
& \leq \int_{0}^{s} \sup _{u \in \Gamma}\left(\sum_{i=0} \frac{\partial \tilde{F}}{\partial z_{i}}\left(\left\langle f_{0}, \nu\right\rangle, \cdots,\left\langle f_{\ell}, \nu\right\rangle\right)\left\langle A(u) f_{i}, \nu\right\rangle\right) d t \\
& =s, \sup _{u \in \Gamma}\left(\sum_{i=0}^{\ell} \frac{\partial \tilde{F}}{\partial z_{i}}\left(\left\langle f_{0}, \nu\right\rangle, \cdots,\left\langle f_{\ell}, \nu\right\rangle\right)\left\langle A(u) f_{i}, \nu\right\rangle\right) \\
& =\sup _{u \in \Gamma} \sum_{i=0}^{\ell} \int_{0}^{s} \frac{\partial \tilde{F}}{\partial z_{i}}\left(\left\langle f_{0}, \nu\right\rangle, \cdots,\left\langle f_{\ell}, \nu\right\rangle\right)\left\langle A(u) f_{i}, \nu\right\rangle d t
\end{aligned}
$$

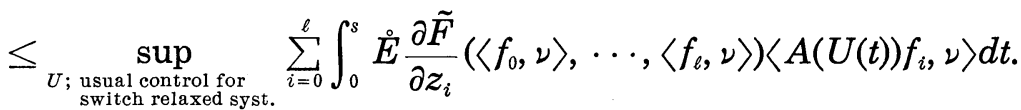


Taking the supremum of left side of (7.21) with respect to $U(t)$ for switching admissible systems, we have

(7.22) $\frac{1}{s} \sup _{U \text {; switch syst. }} \sum_{i=0}^{\ell} \int_{0}^{s} \dot{E} \frac{\partial \tilde{F}}{\partial z_{i}}\left(\left\langle f_{0}, \nu\right\rangle, \cdots,\left\langle f_{\ell}, \nu\right\rangle\right)\left\langle A(U(t)) f_{i}, \nu\right\rangle d t=G \Phi(\nu)$.

On the other hand $(7.17) \sim(7.19)$ tell us that

$$
\begin{aligned}
& \mid \stackrel{\circ}{E} \Phi\left(\Lambda\left(s, Y_{\mathscr{s}}, q_{\mathscr{s}}, \nu\right)\right)-\Phi(\nu)-\int_{0}^{s} \sum_{i=0}^{\ell} \frac{\circ}{E} \frac{\partial \tilde{F}}{\partial z_{j}}\left(\left\langle f_{0}, \nu\right\rangle, \cdots,\left\langle f_{\ell}, \nu\right\rangle\right)\left\langle A(U(t)) f_{i}, \nu\right\rangle \\
& \quad+\frac{1}{2} \sum_{i j=0}^{\ell} \frac{\partial^{2} \tilde{F}}{\partial z_{i} \partial z_{j}}\left(\left\langle f_{0}, \nu\right\rangle, \cdots,\left\langle f_{\ell}, \nu\right\rangle\right)\left\langle h f_{i}, \nu\right\rangle\left\langle h f_{j}, \nu\right\rangle d t \mid \\
& \quad \leq 2 K_{5}(n+1)^{2}\|\nu\| s^{3 / 2} .
\end{aligned}
$$

Appealing to (7.22) we have

$$
\left|\frac{1}{s}(S(s) \Phi(\nu)-\Phi(\nu))-G \Phi(\nu)\right| \leq 2 K_{5}(n+1)^{2}\|\nu\| s^{1 / 2}
$$

Recalling (5.16) we have

$$
\left\|\Lambda\left(s, Y_{s}, q_{s}, \nu\right)\right\| \geq\|\nu\| e^{-K_{1}(a+1)^{2}(s+1)} \quad \text { whenever } \sup _{t \leq s}\left|Y_{s}(t)\right| \leq a .
$$

Since $Y_{\star}$ is a Brownian motion, a martingale inequality implies

$$
\stackrel{\circ}{P}\left(\sup _{t \leq s}\left|Y_{\mathscr{A}}(t)\right| \geq a\right) \leq \frac{s}{a^{2}} .
$$

Putting $a=1 / \sqrt{\varepsilon}$ and $\tilde{N}(\varepsilon)=(N+1) e^{K_{1}(a+1)^{2}(s+1)}$, we see, from (7.25) and

$$
\begin{gathered}
\stackrel{\circ}{P}\left(\left\|\Lambda\left(s, Y_{\infty}, q_{\mathscr{s}}, \nu\right)\right\| \leq N+1\right) \leq \stackrel{\circ}{P}\left(\sup _{t \leq s}\left|Y_{\infty}(t)\right|>\frac{1}{\sqrt{\varepsilon}}\right)<\varepsilon s \\
\text { whenever }\|\nu\| \geq \tilde{N}(\varepsilon) .
\end{gathered}
$$

Therefore, if $\|\nu\| \geq \tilde{N}(\varepsilon)$, then

$$
E\left|\Phi\left(\Lambda\left(s, Y_{s}, q_{\mathscr{s}}, \nu\right)\right)\right| \leq\|\Phi\| \varepsilon s .
$$

This implies, by virtue of “ $\Phi(\nu)=G \Phi(\nu)=0$ for $\|\nu\| \geq \tilde{N}(\varepsilon)$ ”,

$$
\frac{1}{s}(S(s) \Phi(\nu)-\Phi(\nu))-G \Phi(\nu) \mid \leq\|\Phi\| \varepsilon, \quad \text { whenever }\|\nu\| \geq \tilde{N}(\varepsilon) .
$$

Appealing to (7.24), we have 


$$
\begin{gathered}
\left|\frac{1}{s}(S(s) \Phi(\nu)-\Phi(\nu))-G \Phi(\nu)\right| \leq(1+\|\Phi\|) \varepsilon, \\
\text { whenever } s \leq \varepsilon /\left(2 K_{5}(n+1)^{2} \tilde{N}(\varepsilon)\right)^{2} .
\end{gathered}
$$

This implies that $1 / s(S(s) \Phi(\nu)-\Phi(\nu))$ converges to $G \Phi(\nu)$ uniformly on $M\left(R^{n}\right)$, as $s \downarrow 0$.

TheOREM 8. $\mathscr{D}(\$) \supset \mathscr{D}$ and

$$
\circlearrowleft S \Phi=G \Phi \text { on } \mathscr{D}
$$

that is, the generator is an extension of $G$.

\section{§8. Time discrete approximation}

First we recall an approximation theorem of Proposition 4.1 and Theorem 4, namely, for $\mathscr{A}=(\Omega, F, \stackrel{\circ}{P}, \xi, B, T, q)$ there exists an approximate usual control $U_{n}$, such that

(8.1) $U_{n}$ is an $\sigma_{t}(q)$-progressively measurable $\Gamma$-valued process

(8.2) $q_{n}(t, A)=\int_{0}^{t} \delta_{U_{n}(s)}(A) d s$ is a switching relaxed control of $\mathscr{A}_{n}=(\Omega$, $\left.F, \stackrel{\circ}{P}, \xi, B, Y, q_{n}\right)$

and

(8.3) $J(t, \mathscr{A}, \nu, \phi)=\lim _{n \rightarrow \infty} J\left(t, \mathscr{A}_{n}, \nu, \phi\right)$ for $\forall \phi \in C$.

Now we define a usual admissible system $\tilde{A}=(\Omega, F, \stackrel{\circ}{P}, \xi, B, Y, U)$ as follows: $(\Omega, F, \stackrel{\circ}{P}, \xi, B, Y$ ) satisfies the same conditions as an admissible (relaxed) system, $U$ is a $\Gamma$-valued process, $\xi, B$ and $(Y, U)$ are independent and $Y$ is a $\sigma_{t}(Y, U)$-Brownian motion.

$\tilde{\mathfrak{U}}$ denotes the totality of usual admissible systems, and we apply similar notations as for the relaxed case. Putting $\tilde{q}_{U}(t, A)=\int_{0}^{t} \delta_{U(s, \omega)}(A) d s$, we see $\mathscr{A}_{U}=\left(\Omega, F, P, \xi, B, Y, \tilde{q}_{U}\right) \in \mathfrak{A}$. Thus a usual admissible system can be regarded as an admissible (relaxed) system. Moreover a unique solution $\tilde{X}$ of the SDE,

$$
\left\{\begin{array}{l}
d \tilde{X}(t)=\alpha(\tilde{X}(t)) d B(t)+\gamma((t), U(t)) d t \\
\tilde{X}(0)=\xi
\end{array}\right.
$$

gives a unique solution $X_{s_{U}}(=\hat{X})$.

Since $(\xi, B)$ and $(Y, U)$ are independent, we can calculate 


$$
\stackrel{\circ}{E}\left(f(\hat{X}(\theta)) L(\theta, \tilde{\mathscr{A}}) / \sigma_{t}(Y, U)\right)
$$

in the same way as (5.8), and get

$$
\begin{aligned}
& \stackrel{\circ}{E}(f(\hat{X}(\theta)) L(\theta, \tilde{\mathscr{A}}) / Y=y, U(t)=v(t) \text { a.a. } t) \\
& \quad=\stackrel{\circ}{E} f\left(\eta_{v}(\theta) \mathscr{L}\left(\theta, \xi, B, y, q_{v}\right)\right. \\
& \quad=\stackrel{\circ}{E}\left(f\left(X_{\mathscr{A}_{U}}(\theta)\right) L\left(\theta, \mathscr{A}_{U}\right) / Y=y, q_{U}=\lambda_{v}\right)
\end{aligned}
$$

where $\lambda_{v}(t, A)=\int_{0}^{t} \delta_{v(s)}(A) d s$. We remark that, if $v(t)=v^{*}(t)$, a.a. $t$, then $\lambda_{v}=\lambda_{v^{*}}$. The unnormalized conditional distribution $\tilde{\Lambda}(t, \tilde{\mathscr{A}}, v)$ is defined by

$$
\langle f, \tilde{\Lambda}(\theta, \tilde{\mathscr{A}}, v)\rangle=\stackrel{\circ}{E}_{\nu}\left\langle f(\hat{X}(\theta)) L(\theta, \tilde{\mathscr{A}}) / \sigma_{t}(Y, U)\right) \quad \text { for } f \in C_{b}\left(R^{n}\right) .
$$

Hence (8.5) implies

(8.7) $\tilde{\Lambda}(\theta, \tilde{\mathscr{A}}, v)=\Lambda\left(\theta, Y, q_{U}, \nu\right), \stackrel{\circ}{P}$-almost surely. We sometimes put $\tilde{\Lambda}(\theta$, $\tilde{\mathscr{A}}, \nu)=\tilde{\Lambda}(\theta, Y, U, \nu)$ and $\tilde{J}(\theta, \tilde{\mathscr{A}}, \nu, \phi)=\stackrel{\circ}{E} \phi(\tilde{\Lambda}(\theta, \tilde{\mathscr{A}}, \nu))=\tilde{J}(\theta, Y, U, \nu, \phi)$.

We approximate $U_{n}$ of (8.1) (say $W$ for simplicity) by a switching usual control, by a routine method, i.e. $\tilde{U}_{k}$ and $\tilde{U}_{k, p}$ are defined as follows

$$
\tilde{U}_{k}(t)=2^{k} \int_{t-2-k}^{t} W(s) d s \quad \text { and } \quad \tilde{U}_{k, p}(t)=\tilde{U}_{k}\left(\frac{\left[2^{p} t\right]}{2^{p}}\right) .
$$

Then $\lim _{k \rightarrow \infty} \lim _{p \rightarrow \infty} \tilde{U}_{k, p}(t)=W$ in $L^{2}\left(\left[\begin{array}{ll}0 & T\end{array}\right] \times \Omega\right)$. This fact implies that there exists an approximate switching usual control $W_{k}$, which is $\sigma_{t}(U)$ progressively measurable and satisfies

$$
\stackrel{\circ}{E} \int_{0}^{T}\left|W_{k}(s)-W(s)\right|^{2} d s \rightarrow 0, \quad \text { as } \quad k \rightarrow \infty
$$

and

$$
\tilde{J}(t, Y, W, \nu, \phi)=\lim _{k \rightarrow \infty} \tilde{J}\left(t, Y, W_{k}, \nu, \phi\right) .
$$

By (8.8) some subsequence of $W_{k}$ converges to $W$ a.e. in $[0 T] \times \Omega$, we assume " $W_{k} \rightarrow W$ a.e." for simplicity. Therefore, for a.a. $\omega(\stackrel{P}{)})$,

$$
\begin{aligned}
& \int_{\Gamma} g(u) \tilde{q}_{W_{k}}(t, d u)=\int_{0}^{t} \int_{\Gamma} g(u) \delta_{W_{k}}(d u) d s \\
& =\int^{t} g\left(W_{k}(s)\right) d s \underset{k \rightarrow \infty}{\longrightarrow} \int_{0}^{t} g(W(s)) d s=\int_{\Gamma} g(u) \tilde{q}_{W}(t, d u) \\
& \quad \text { for any } t \text { and } g \in C_{b}(\Gamma) .
\end{aligned}
$$

This implies, as $k \rightarrow \infty$. 


$$
\hat{d}_{T}\left(\tilde{q}_{W_{k}}, \tilde{q}_{W}\right) \rightarrow 0, \quad \stackrel{\circ}{P} \text {-almost surely } .
$$

Hence by Propositions 2.2 and the Corollary of Proposition 3.1 we see

$$
\mathscr{A}_{W_{k}} \rightarrow \mathscr{A}_{W} \text { and }\left(X_{W_{k}}, \mathscr{A}_{W k}\right) \rightarrow\left(X_{W}, \mathscr{A}_{W}\right) \text { in } D_{T} \text {. }
$$

Therefore Theorem 6 implies that, for $\forall \theta$,

$$
\tilde{\Lambda}\left(\theta, Y, W_{k}, \nu\right) \rightarrow \tilde{\Lambda}(\theta, Y, W, \nu) \quad \text { in metric } \delta .
$$

So we have

Proposition 8.1. For $\mathscr{A}=(\Omega, F, P, \xi, B, Y, q)$ there exists a switching usual control $W_{k}, k=1,2, \cdots$, which is $\sigma_{t}(q)$-progressively measurable and for any $\theta$

$$
\tilde{\Lambda}\left(\theta, Y, W_{k}, \nu\right) \rightarrow \Lambda(\theta, Y, q, \nu) \quad \text { in metric } \delta \text {, as } k \rightarrow \infty .
$$

This means that switching usual controls are rich enough in the class of relaxed controls.

Put $\widetilde{\mathfrak{A}}_{N}=$ totality of usual admissible systems whose usual controls are switching with time interval $2^{-N}$, i.e. $\tilde{\mathscr{A}}=(\Omega, F, \stackrel{\mathscr{P}}{\xi}, B, Y, U) \in \widetilde{\mathfrak{A}}_{N}$, iff $U(t)=U\left(\left[2^{N} t\right] / 2^{N}\right)$. We denote $\tilde{\mathscr{A}} \in \widetilde{\mathfrak{A}}_{0}$, if $U$ is constant control. So $\widetilde{\mathfrak{A}}_{0} \subset \widetilde{\mathfrak{A}}_{N}$. When $P_{\xi}=\nu /\|\nu\|$, we say $\tilde{\mathscr{A}} \in \widetilde{\mathfrak{A}}_{N}(\nu /\|\nu\|)$. Put $\widetilde{\mathfrak{A}}=\bigcup_{N=0}^{\infty} \widetilde{\mathfrak{A}}_{N}$.

From Proposition 8.1 we see

$$
\begin{aligned}
S(t) \phi(\nu) & =\sup _{\mathscr{A} \in \tilde{\mathscr{A}}(\nu /\|\nu\|)} \tilde{J}(t, \tilde{\mathscr{A}}, \nu, \phi) \\
& =\lim _{N \rightarrow \infty} \sup _{\tilde{\mathscr{A}} \in \tilde{\mathfrak{U}}_{N}(\nu /\|\nu\|)} \tilde{J}(t, \tilde{\mathscr{A}}, \nu, \phi) .
\end{aligned}
$$

Define $Q=Q_{N}$ by

$$
Q \phi(\nu)=\sup _{\mathscr{z} \in \tilde{\mathscr{U}}_{N}(\nu /\|\nu\|)} J\left(2^{-N}, \tilde{\mathscr{A}}, \nu, \phi\right)
$$

We remark that

$$
\begin{aligned}
& J\left(2^{-N}, \tilde{\mathscr{A}}, \nu, \phi\right)=\stackrel{\circ}{E} \phi\left(\tilde{\Lambda}\left(2^{-N}, Y, U(0), \nu\right)\right) \\
& \quad=\int_{\Gamma} \stackrel{\circ}{E}\left(\phi\left(\tilde{\Lambda}\left(2^{-N}, Y, U(0), \nu\right)\right) / U(0)=u\right) P_{U_{0}}(d u) .
\end{aligned}
$$

Since $(Y(\theta), \theta \geq 0)$ is independent of $U(0)$,

$$
\stackrel{\circ}{E}\left(\phi\left(\tilde{\Lambda}\left(2^{-N}, Y, U(0), \nu / U(0)=u\right)\right)\right)=\tilde{J}\left(2^{-N}, Y, u, \nu, \phi\right) .
$$

Moreover the value of the left side depends only on $N, u, \nu, \phi$, since $Y$ is a Brownian motion with respect to $\stackrel{P}{P}(\cdot / U(0)=u)$. We put 


$$
\tilde{J}\left(2^{-N}, Y, u, \nu, \phi\right)=\Phi_{N}(u, \nu ; \phi)
$$

and

$$
\tilde{Q} \phi(\nu)=\sup _{u \in \Gamma} \Phi_{N}(u, \nu, \phi)
$$

Then $\Phi_{N}(u, \nu, \phi)$ is continuous in $(u, \nu) \in \Gamma \times\left(M\left(R^{n}\right), \Delta\right)$ and $\tilde{J}\left(2^{-N}, Y, u, \nu, \phi\right)$ $\leq \tilde{Q} \phi(\nu)$. Combining with (8.12), we see

$$
\begin{aligned}
Q \phi(\nu) & \leq \tilde{Q} \phi(\nu)=\sup _{\tilde{\mathscr{A}} \in \tilde{U}_{0}(\nu /\|\nu\|)} \tilde{J}\left(2^{-N}, \tilde{\mathscr{A}}, \nu, \phi\right) \\
& \leq \sup _{\tilde{\mathscr{A}} \in \tilde{\mathscr{U}}_{N}(\nu /\|\nu\|)} \tilde{J}\left(2^{-N}, \tilde{\mathscr{A}}, \nu, \phi\right)=Q \phi(\nu) .
\end{aligned}
$$

Hence we have

$$
Q_{N}=\tilde{Q}
$$

Since $\Gamma$ is compact, using a measurable selection theorem, we can take a Borel measurable mapping, $v=v_{\phi}: M\left(R^{n}\right) \rightarrow \Gamma$ such that

$$
\left.\Phi_{N}\left(v_{\phi}(\nu), \nu, \phi\right)\right)=\sup _{u \in \Gamma} \Phi_{N}(u, \nu, \phi) .
$$

This gives

$$
Q \phi(\nu)=\tilde{J}\left(2^{-N}, Y, v_{\phi}(\nu), \nu, \phi\right)
$$

We have, for $\tilde{\mathscr{A}} \in \widetilde{\mathfrak{A}}_{N}(\nu /\|\nu\|)$

$$
\begin{aligned}
J\left(2^{-N+1}, \tilde{\mathscr{A}}, \nu, \phi\right)=\stackrel{\circ}{E} \phi\left(\tilde{\Lambda}\left(2^{-N+1}, Y, U, \nu\right)\right) \\
=\stackrel{\circ}{E} \phi\left(\tilde{\Lambda}\left(2^{-N}, Y_{2^{+}-}, U_{2^{+}-N}^{+}, \tilde{\Lambda}\left(2^{-N}, Y, U, \nu\right)\right)\right) \\
\leq(Q \phi)\left(\tilde{\Lambda}\left(2^{-N}, Y, U, \nu\right)\right) \leqq Q(Q \phi)(\nu) \\
\quad=Q^{2} \phi(\nu) .
\end{aligned}
$$

Define $U_{2}=U_{2, \phi, \nu}$ by

$$
U_{2}(t)=\left\{\begin{array}{l}
v_{Q \phi}(\nu), 0 \leq t \leq 2^{-N} \\
v_{\phi}\left(\tilde{\Lambda}\left(2^{-N}, Y, v_{Q \phi}(\nu), \nu\right)\right), 2^{-N}<t .
\end{array}\right.
$$

Then $\mathscr{A}^{*}=\left(\Omega, F, \stackrel{\circ}{P}, \xi, B, Y, U_{2}\right) \in \widetilde{\mathfrak{A}}_{N}(\nu /\|\nu\|)$ and

$$
\begin{aligned}
& J\left(2^{-N+1}, \mathscr{A}^{*}, \nu, \phi\right)=\stackrel{\circ}{E} \phi\left(\tilde{\Lambda}\left(2^{-N+1}, Y, U_{2}, \nu\right)\right) \\
& =\stackrel{\circ}{E} \stackrel{\circ}{E}\left(\phi \left(\tilde { \Lambda } \left(2^{-N}, Y_{2^{-}}^{+}, v_{\phi}\left(\tilde{\Lambda}\left(2^{-N}, Y, v_{Q \phi}(\nu), \nu\right)\right),\right.\right.\right. \\
& \left.\left.\left.\quad \tilde{\Lambda}\left(2^{-N}, Y, v_{Q \phi}(\nu), \nu\right)\right) / \sigma_{2-N}(Y)\right)\right) \\
& =\stackrel{\circ}{E}(Q \phi)\left(\tilde{\Lambda}\left(2^{-N}, Y, v_{Q \phi}(\nu), \nu\right)\right) \\
& =Q(Q \phi)(\nu)=Q^{2} \phi(\nu) .
\end{aligned}
$$


Combining with (8.24), we have

$$
\begin{aligned}
Q^{2} \phi(\nu) & =\sup _{\mathscr{A} \in \tilde{\mathscr{U}}_{N}(\nu /\|\nu\| \|)} \tilde{J}\left(2^{-N+1}, \tilde{\mathscr{A}}, \nu, \phi\right) \\
& =\tilde{J}\left(2^{-N+1}, \mathscr{A}^{*}, \nu, \phi\right) .
\end{aligned}
$$

Repeating a similar calculation we see

$$
Q^{k+1} \phi(\nu)=\sup _{\mathscr{A} \in \tilde{\mathscr{U}}_{N}(\nu /\|\nu\|)} \tilde{J}\left((k+1) 2^{-N}, \tilde{\mathscr{A}}, \nu, \phi\right)
$$

and an optimal one $U_{k+1}=U_{k+1, \nu, \phi}$ is given successively by

$$
U_{k+1}(t)=\left\{\begin{array}{l}
U_{k, \nu, Q^{k_{\phi}}}(t), \quad 0 \leq t \leq k 2^{-N} \\
v_{\phi}\left(\tilde{\Lambda}\left(k 2^{-N}, Y, U_{k, \nu, Q^{k} \phi}, \nu\right), \quad k 2^{-N}<t .\right.
\end{array}\right.
$$

Recalling (8.14) we see, for binary $t$ (say $j 2^{-p}$ )

$$
\lim _{\nu \rightarrow \infty} \sup _{\tilde{A} \in \tilde{H}_{N}(\nu /\|\nu\|)} \tilde{J}(t, \tilde{\mathscr{A}}, \nu, \phi)=S(t) \phi(\nu)=\lim _{N \rightarrow \infty} Q_{N}^{\left[2^{N} t\right]} \phi(\nu)
$$

and an approximate optimal usual switching control is given by $U_{k, \nu, \phi}$.

\section{REFERENCES}

[1] A. Bensoussan and J. L. Lions, Application des inéquations variationnelles en contrôle stochastique, Dunod, Paris 1978.

[2] P. Billingsley, Convergence of Probability Measures, John Wiley 1968.

[ 3 ] J. M. Bismut, Partially observed diffusions and their control, SIAM. J. Control Optim., 20 (1982), 302-309.

[4] M. H. A. Davis and M. Kohlmann, On the nonlinear semigroup of stochastic control under partial observations, preprint.

[5] W. H. Fleming, Generalized solutions in optimal stochastic control, Differential games and control theory II, Lect. Notes in pure and Appl. math., 30, Dekker.

[6] —- Nonlinear semigroup for controlled partially observed diffusions, SIAM. J. Control Optim., 20 (1982), 286-301.

[ 7 ] — and M. Nisio, On the existence of optimal stochastic controls, J. Math. Mech., 15 (1966), 777-794.

[ 8 ] - and E. Pardoux, Optimal control for partially observed diffusions, SIAM. J. Control Optim., 20 (1982), 258-285.

[9] U. G. Haussman, On the existence of optimal controls for partially observed diffusions, SIAM. J. Control Optim., 20 (1982), 385-407.

[10] M. Nisio, Stochastic Control Theory, ISI Lect. Note 9, McMillan India, 1981.

[11] K. R. Parthasarathy, Probability Measures on Metric Spaces, Acad. Press, 1967.

[12] A. V. Skorobod, Studies in the Theory of Random Processes, Addism-Wesly, 1965 (orig. Russian). 
W. H. Fleming

Division of Applied Mathematics

Brown University

Prividence R. I.

USA

M. Nisio

Department of Mathematics

Kobe University

Rokko, Kobe 657

Japan 